\title{
A PROOF OF ANDREWS' CONJECTURE ON PARTITIONS WITH NO SHORT SEQUENCES
}

\author{
DANIEL M. KANE ${ }^{1}$ and ROBERT C. RHOADES ${ }^{2}$ \\ ${ }^{1}$ University of California, San Diego, 9500 Gilman Drive \#0404, La Jolla, CA 92093-0404; \\ email:dakane@math.ucsd.edu \\ ${ }^{2}$ Susquehanna International Group, Bala Cynwyd, PA 19004; \\ email: rob.rhoades@gmail.com
}

Received 10 March 2017; accepted 5 February 2019

\begin{abstract}
Our main result establishes Andrews' conjecture for the asymptotic of the generating function for the number of integer partitions of $n$ without $k$ consecutive parts. The methods we develop are applicable in obtaining asymptotics for stochastic processes that avoid patterns; as a result they yield asymptotics for the number of partitions that avoid patterns.

Holroyd, Liggett, and Romik, in connection with certain bootstrap percolation models, introduced the study of partitions without $k$ consecutive parts. Andrews showed that when $k=2$, the generating function for these partitions is a mixed-mock modular form and, thus, has modularity properties which can be utilized in the study of this generating function. For $k>2$, the asymptotic properties of the generating functions have proved more difficult to obtain. Using $q$-series identities and the $k=2$ case as evidence, Andrews stated a conjecture for the asymptotic behavior. Extensive computational evidence for the conjecture in the case $k=3$ was given by Zagier.

This paper improved upon early approaches to this problem by identifying and overcoming two sources of error. Since the writing of this paper, a more precise asymptotic result was established by Bringmann, Kane, Parry, and Rhoades. That approach uses very different methods.
\end{abstract}

2010 Mathematics Subject Classification: 05A17, 11P82, 60C05

\section{Introduction and statement of results}

Studying a generalization of bootstrap percolation (see $[1,4,14,16]$ for examples), Holroyd, Liggett, and Romik [17] introduced the following probability

(C) The Author(s) 2019. This is an Open Access article, distributed under the terms of the Creative Commons Attribution licence (http://creativecommons.org/licenses/by/4.0/), which permits unrestricted re-use, distribution, and reproduction in any medium, provided the original work is properly cited. 
models: let $0<s<1$ and $C_{1}, C_{2}, \ldots$ be independent events with probabilities

$$
\mathbf{P}_{s}\left(C_{n}\right):=1-e^{-n s}
$$

under a probability measure $\mathbf{P}_{s}$. Let $A_{k}$ be the event

$$
A_{k}=\bigcap_{i=1}^{\infty}\left(C_{i} \cup C_{i+1} \cup \cdots \cup C_{i+k-1}\right)
$$

that there is no sequence of $k$ consecutive $C_{i}$ values that do not occur. The relevant question in [17] is to understand the behavior as $s \downarrow 0$. Theorem 2 of Holroyd, Liggett, and Romik [17] gives

$$
\log \left(\mathbf{P}_{s}\left(A_{k}\right)\right) \sim-\frac{L_{k}}{s}
$$

where

$$
L_{k}:=\frac{\pi^{2}}{3 k(k+1)} .
$$

Obtaining an estimate for $\mathbf{P}_{s}\left(A_{k}\right)$ with polynomial relative error has proven to be a challenging problem. Bringmann and Mahlburg [9] refined the result of Holroyd, Liggett, and Romik by proving nonlogarithmic lower and upper bounds that differed by a polynomial factor of $s^{-1 / k}$. Precisely, they give [9, Theorem 1.2]

$$
\exp \left(-\frac{L_{k}}{s}\right) \ll_{k} \mathbf{P}_{s}\left(A_{k}\right) \ll_{k} s^{-(2 k-1) / 2 k} \exp \left(-\frac{L_{k}}{s}\right) .
$$

With Mellit, Bringmann and Malburg [10] developed a general method for establishing similar bounds for natural families of pattern-avoiding sequences. Underlying all of the above results are estimates for the eigenvalues of an associated (Markov-type) stochastic process.

Prior to the asymptotic results of Bringmann, Mahlburg, and Mellit, Andrews [3] established a surprising connection between $\mathbf{P}_{s}\left(A_{2}\right)$ and one of Ramanujan's mock theta functions. Precisely, he showed

$$
\mathbf{P}_{s}\left(A_{2}\right)=\prod_{n=1}^{\infty} \frac{1+q^{3 n}}{1+q^{n}} \cdot \chi(q),
$$

where $q:=e^{-s}$ and $\chi(q)=\sum_{n=0}^{\infty} q^{n^{2}} \prod_{m=1}^{n}\left(\left(1+q^{m}\right) /\left(1+q^{3 m}\right)\right)$ is a mock theta function. Zwegers' Ph.D. thesis [24] yields the modular properties of Ramanujan's mock theta functions (see [22] or [21] for details). Consequentially, 
Andrews [3], using (1.2) and additional identities for Ramanujan's mock theta functions proved that

$$
\mathbf{P}_{s}\left(A_{2}\right) \sim \sqrt{\frac{\pi}{2}} s^{-1 / 2} \exp \left(-\frac{\pi^{2}}{18 s}\right) \quad \text { as } s \downarrow 0 .
$$

Using additional $q$-series identities when $k>2$, he made the following conjecture.

CONJECTURE 1.1 (Andrews [3]). For each $k \geqslant 2$, there exists a positive constant $D_{k}$ such that

$$
\mathbf{P}_{s}\left(A_{k}\right) \sim D_{k} s^{-1 / 2} \exp \left(-\frac{\lambda_{k}}{s}\right) \quad \text { as } s \downarrow 0 .
$$

We prove the following precise version of Andrews' conjecture.

THEOREM 1.2. Andrews' conjecture is true with $D_{k}=\sqrt{2 \pi} / k$. More specifically, we have

$$
\mathbf{P}_{s}\left(A_{k}\right)=\frac{\sqrt{2 \pi}}{k} s^{-1 / 2} \exp \left(-\frac{\pi^{2}}{3 k(k+1) s}+O_{k}\left(s^{1 /(2 k+3)}\right)\right) .
$$

REMARK 1.3. We expect that our techniques can be improved to give a full asymptotic expansion for $\mathbf{P}_{s}\left(A_{k}\right)$ with relative error $O\left(s^{N}\right)$, for any $N$. Since the writing of this paper, such an asymptotic expansion was established by Bringamnn, Kane, Parry, and Rhoades [7]. The methods are very different relying on asymptotic expansions of Wright's generalization of the Bessel function.

1.1. More on partitions and additional applications. There is an unexpected and beautiful connection between bootstrap percolation models and partitions, Ramanujan's mock theta functions, and the Rogers-Ramanujan identities.

A partition $\mu$ of $n$ has a $k$-sequence if there are $k$ parts of consecutive sizes. Let $p_{k}(n)$ denote the number of partitions of $n$ with no $k$-sequences and $G_{k}(q):=$ $\sum_{n=0}^{\infty} p_{k}(n) q^{n}$ the generating function. Set $p_{k}(0)=1$. In [17, Section 4] it is shown that

$$
\mathbf{P}_{s}\left(A_{k}\right)=\frac{G_{k}(q)}{P(q)}
$$

where $q:=e^{-s}$ and $P(q)=\sum_{n=0}^{\infty} p(n) q^{n}=\prod_{n=1}^{\infty}\left(1 /\left(1-q^{n}\right)\right)$, is the generating function for $p(n)$, the number of partitions of $n$. 
Asymptotics for $P(q)$ are well known, namely

$$
P(q)=\frac{1}{\sqrt{2 \pi}} s^{1 / 2} \exp \left(\frac{\pi^{2}}{6 s}-\frac{s}{24}+O\left(s^{N}\right)\right)
$$

for any $N$. Thus, by (1.3), determination of the asymptotics of $G_{k}(q)$ is equivalent to the determination of the asymptotic of $\mathbf{P}_{s}\left(A_{k}\right)$. We prove the following theorem, which is equivalent to Theorem 1.2.

THEOREM 1.4. For each $k \geqslant 2$ we have

$$
G_{k}\left(e^{-s}\right)=\frac{1}{k} \exp \left(\frac{\pi^{2}}{6 s}\left(1-\frac{2}{k(k+1)}\right)+O_{k}\left(s^{1 /(2 k+3)}\right)\right)
$$

as $s \downarrow 0$.

REMARK 1.5. A slight modification of the arguments presented establish Theorem 1.4 with a relative error that is $o(1)$ for nonreal $s$ satisfying $|\Im(s)|=o(\Re(s))$.

Exploiting the connection between $G_{k}\left(e^{-s}\right)$ and $\mathbf{P}_{s}\left(A_{k}\right)$, a more refined version of Conjecture 1.1 is the following.

CONJECTURe 1.6. For $s$ real and $s \downarrow 0$

$$
G_{k}\left(e^{-s}\right)=\frac{1}{k} \exp \left(\frac{\pi^{2}}{6 s}\left(1-\frac{2}{k(k+1)}\right)+\alpha_{k} s^{1 / k}+O\left(s^{2 / k}\right)\right)
$$

for some constant $\alpha_{k}$.

Classical results on the mock theta functions [3] gives this conjecture for $k=2$ with $\alpha_{2}=\sqrt{2 / 9 \pi}$. Using a numerical technique, Zagier [23] calculated that $\alpha_{3} \approx 0.26627104041 \ldots$, which he conjectured was $3^{1 / 3} / 4 \Gamma(2 / 3)$. As previously mentioned, this stronger conjecture was established in [7].

REMARK 1.7. This conjecture implies that for $k>2$, the generating function $G_{k}(q)$ is not a usual modular form. Indeed, if $G_{k}(q)$ is a half integral weight modular form or mixed-mock modular form, we would expect an asymptotic expansion that contains only powers of $s^{1 / 2}$.

It is well known that the asymptotic behavior of generating functions leads to asymptotics for the coefficients. We obtain the following theorem for the asymptotic of $p_{k}(n)$. 
THEOREM 1.8. As $n \rightarrow \infty$ we have

$$
p_{k}(n) \sim \frac{1}{2 k}\left(\frac{1}{6}\left(1-\frac{2}{k(k+1)}\right)\right)^{1 / 4} \frac{1}{n^{3 / 4}} \exp \left(\pi \sqrt{\frac{2}{3}\left(1-\frac{2}{k(k+1)}\right) n}\right) .
$$

REMARK 1.9. Bringmann and Mahlburg [8] use the connection with Ramanujan's mock theta function and an extension of the circle method to prove a nearly exact formula for $p_{2}(n)$.

While the study of partitions without $k$-sequences for $k>2$ is relatively new, there are several classical results on partitions without 2-sequences. The RogersRamanujan identities state that

$$
\begin{aligned}
& \sum_{n=0}^{\infty} \frac{q^{n^{2}}}{(1-q) \cdots\left(1-q^{n}\right)}=\prod_{n=1}^{\infty} \frac{1}{\left(1-q^{5 n-4}\right)\left(1-q^{5 n-1}\right)}, \\
& \sum_{n=0}^{\infty} \frac{q^{n^{2}+n}}{(1-q) \cdots\left(1-q^{n}\right)}=\prod_{n=1}^{\infty} \frac{1}{\left(1-q^{5 n-3}\right)\left(1-q^{5 n-2}\right)} .
\end{aligned}
$$

MacMahon [20] found a combinatorial interpretation of the Rogers-Ramanujan identities as a way of counting partitions without 2-sequences with some particular constraints. In particular, he shows that they imply:

(1.4)* The partitions of $n$ into distinct parts with no parts of consecutive size are equinumerous with the number of partitions of $n$ into parts of the form $5 n-4$ and $5 n-1$.

$(1.5)^{*}$ The partitions of $n$ into distinct parts with no parts of consecutive size and no parts of size 1 are equinumerous with the number of partitions of $n$ into parts of the form $5 n-3$ and $5 n-2$.

This combinatorial interpretation is important in the hard hexagon model studied by Baxter [6]. Another use of these identities is that the product expansions reveal that these series in question are essentially modular forms and, thus, their analytic nature is well understood. For example, with $q=e^{-s}$, for any $N$, the series in (1.4) satisfies

$$
\sum_{n=0}^{\infty} \frac{q^{n^{2}}}{(1-q) \cdots\left(1-q^{n}\right)}=\sqrt{\frac{2}{5-\sqrt{5}}} \exp \left(\frac{\pi^{2}}{15 s}-\frac{s}{60}\right)+O\left(s^{N}\right) \quad \text { as } s \downarrow 0 .
$$

It is surprising that the generating functions for partitions without consecutive parts occasionally have product expansions resembling those of the 
Rogers-Ramanujan identities. Let $p_{k, r,>B}(n)$ be the number of partitions of $n$ with no $k$ parts of consecutive sizes, no part occurring more than $r$ times, and no parts of size $\leqslant B$. Then (1.4) and (1.5) are identities for the generating functions $\sum_{n=0}^{\infty} p_{2,1,>0}(n) q^{n}$ and $\sum_{n=0}^{\infty} p_{2,1,>1}(n) q^{n}$. We have the following partition identities:

$$
\begin{aligned}
\sum_{n=0}^{\infty} p_{2,2,>1}(n) q^{n} & =\prod_{n=1}^{\infty} \frac{1}{\left(1-q^{6 n-2}\right)\left(1-q^{6 n-3}\right)\left(1-q^{6 n-4}\right)} \\
\sum_{n=0}^{\infty} p_{2,2,>0}(n) q^{n} & =\prod_{n=1}^{\infty} \frac{\left(1-q^{6 n-3}\right)^{2}\left(1-q^{6 n}\right)}{\left(1-q^{n}\right)} \\
\sum_{n=0}^{\infty} p_{2, \infty,>1}(n) q^{n} & =\prod_{n=1}^{\infty} \frac{1}{\left(1-q^{6 n}\right)\left(1-q^{6 n-2}\right)\left(1-q^{6 n-3}\right)\left(1-q^{6 n-4}\right)}
\end{aligned}
$$

The first and second identities are due to Andrews [2] and the final identity is due to MacMahon [20]; see also Andrews and Lewis [5]. The above identities along with modular form techniques allow for very precise asymptotics for the generating functions of partitions that avoid 2-sequences in addition to satisfying some additional constraints. Asymptotics also exist in some cases where there is no modular relationship. For instance, the case of $p_{k, 1,>0}(n)$ are studied in [11].

Due to (1.3) studying $G_{k}(q)$ is equivalent to studying $\mathbf{P}_{s}\left(A_{k}\right)$ when $q=e^{-s}$. This equivalence provides two equivalent languages in which to discuss our results, that of partitions and that of probability. Throughout most of this paper we will use the former language to discuss our techniques. There are three reasons for this somewhat arbitrary choice. First, the discussion of the behavior of the small parts of the partition (or equivalently the $C_{i}$ for small $i$ ) fits slightly more naturally into this language. Second, our own backgrounds are in combinatorics. Finally, numerical calculations suggest the asymptotics of the function $G_{k}\left(e^{-s}\right)$ is given purely in powers of $s^{1 / k}$, whereas the asymptotics of $\mathbf{P}_{s}\left(A_{k}\right)$ will have an extra $s^{1 / 2}$ multiplying all terms.

1.2. The approach. In this section, we sketch the proof of Andrews' conjecture. The fundamental idea is to compute $G_{k}(q)$ as the limiting value of a recurrence relation. In particular, one can imagine building a partition by adding parts of one size at a time: first determining the number of parts of size one, then the number of parts of size two and so on. In order to ensure that the partition constructed has no $k$-sequences, one would need to keep track of how many of the recent part sizes have been used and ensure that no $k$ sizes in a row are employed. In order to keep track of the necessary generating functions, 
we define

$$
\widetilde{v}_{i}^{k}(N, q):=\sum_{\begin{array}{c}
\mu \text { a partition with parts } \leqslant N \\
\mu \text { has no } k \text { parts with consecutive sizes } \\
\mu \text { has parts of size } N, N-1, \ldots, N-i+1 \\
\mu \text { has no part of size } N-i
\end{array}} q^{|\mu|} .
$$

In particular, we note that for $N=0$ we have that

$$
\widetilde{v}_{i}^{k}(0, q)= \begin{cases}1 & \text { if } i=0, \\ 0 & \text { otherwise }\end{cases}
$$

We have the following recursion

$$
\left(\begin{array}{c}
\widetilde{v}_{0}^{k}(N, q) \\
\widetilde{v}_{1}^{k}(N, q) \\
\vdots \\
\widetilde{v}_{k-1}^{k}(N, q)
\end{array}\right)=\left(\begin{array}{cccc}
1 & 1 & \cdots & 1 \\
z\left(q^{N}\right) & 0 & \cdots & 0 \\
0 & z\left(q^{N}\right) & \cdots & 0 \\
0 & & \vdots & 0 \\
0 & \cdots & z\left(q^{N}\right) & 0
\end{array}\right)\left(\begin{array}{c}
\widetilde{v}_{0}^{k}(N-1, q) \\
\widetilde{v}_{1}^{k}(N-1, q) \\
\vdots \\
\widetilde{v}_{k-1}^{k}(N-1, q)
\end{array}\right)
$$

where $z(x):=x /(1-x)$. For convenience set

$$
m(x):=\left(\begin{array}{cccc}
1 & 1 & \cdots & 1 \\
z(x) & 0 & \cdots & 0 \\
0 & z(x) & \cdots & 0 \\
0 & & \vdots & 0 \\
0 & \cdots & z(x) & 0
\end{array}\right)
$$

and

$$
v^{k}(N, q):=\left(\begin{array}{c}
\widetilde{v}_{0}^{k}(N, q) \\
\widetilde{v}_{1}^{k}(N, q) \\
\vdots \\
\widetilde{v}_{k-1}^{k}(N, q)
\end{array}\right) .
$$

Thus, we have the recursion

$$
v^{k}(N, q)=m\left(q^{N}\right) v^{k}(N-1, q) .
$$

Furthermore, it is not hard to see that

$$
G_{k}(q)=\lim _{N \rightarrow \infty} \widetilde{v}_{0}^{k}(N, q) .
$$

It should also be noted that the truncated generating function $G_{k}(N, q)$ for partitions with parts of size at most $N$ and no $k$-sequences can be obtained as

$$
G_{k}(N, q)=(1,1, \ldots, 1) \cdot v^{k}(N, q) .
$$


Thus, we have a linear, homogeneous recurrence relation with nonconstant coefficients whose limiting value yields $G_{k}(q)$. The main idea for evaluating this quantity is as follows. If the matrices, $m\left(q^{N}\right)$, were constant (or even merely simultaneously diagonalizable), the product would be easy to evaluate and $G_{k}(q)$ would be approximately equal to the product of the largest eigenvalues. This is not the case, but fortunately, the matrices $m\left(q^{n}\right)$ vary slowly with $n$. The difficulty in approximating $G_{k}(q)$ comes in figuring out how to take advantage of this.

This basic approach is not new to this problem. Holroyd, Liggett, and Romik [17] implicitly employ a similar recurrence relation to obtain $\mathbf{P}_{s}\left(A_{k}\right)$. Since the $m\left(q^{n}\right)$ are slowly varying in $n$, they approximate products of roughly $s^{-1 / 2}$ of these matrices by making the approximation that all of the matrices in the block are the same. Within each of these blocks, standard eigenvalue techniques are used to evaluate the product. This technique allows for asymptotic approximation of $\log \left(\mathbf{P}_{s}\left(A_{k}\right)\right)$, yielding a term coming from the product of the largest eigenvalues of $m\left(q^{n}\right)$, but has two major sources of error. The first of these errors comes from the approximation that each of the $m\left(q^{n}\right)$ within a block are constant. This is especially problematic for the early blocks, for which $m\left(q^{n}\right)$ is rapidly varying with $n$. The second source of error comes from having poor control over the transitions between blocks. Our technique avoids these difficulties, but requires new ideas to approximate this product of noncommuting matrices.

Our main idea is to write the vectors $v^{k}(N, q)$ in terms of the slowly varying eigenbasis of the matrices $m\left(q^{n}\right)$. In particular, we may diagonalize each matrix as

$$
m\left(q^{n}\right)=A\left(q^{n}\right) D\left(q^{n}\right) A\left(q^{n}\right)^{-1},
$$

where $D\left(q^{n}\right)$ is the diagonal matrix with $\lambda_{1}\left(q^{n}\right)$, the primary eigenvalue of $m\left(q^{n}\right)$, in the upper-left-hand corner of the matrix. Thus, in the appropriate basis, multiplying by $m$, corresponds to multiplication by the diagonal matrix $D$. Unfortunately, in order to rewrite $v^{k}(n, q)$ in terms of the appropriate eigenbasis for $m\left(q^{n+1}\right)$, we must also multiply by the transition matrix

$$
T(n, q):=A\left(q^{n+1}\right)^{-1} A\left(q^{n}\right) .
$$

Since the coefficients of $A\left(q^{n}\right)$ are slowly varying, $T(n, q) \approx I_{k}$, where $I_{k}$ is the $k \times k$ identity matrix. In particular, with $q=e^{-s}$ we establish $T(n, q)=$ $I_{k}+O\left(n^{-1}+s\right)$. As $n$ becomes large, the primary eigenvalue becomes much larger than the others and so multiplying by $D\left(q^{n}\right)$ decreases the sizes of the other coordinates relative to the first coordinate. Consequentially, the vector of interest is well approximated by the first coordinate. Ignoring the off-diagonal entries of the $T(n, q)$, we find that $G(q)$ is roughly

$$
\prod_{n} \lambda_{1}\left(q^{n}\right) \prod_{n} T(n, q)^{1,1}
$$


where $T(n, q)^{1,1}$ is the upper-left-hand entry of $T(n, q)$. The product $\prod_{n} \lambda_{1}\left(q^{n}\right)$ is handled through an analysis of the characteristic polynomial of $m\left(q^{n}\right)$ by Holroyd, Liggett, and Romik [17]. However, we require a refinement of their calculations to obtain sufficient errors (see Theorem 5.1). The product of the transition matrix entries is similar to, but more delicate than, the analysis used to compute $\prod \lambda_{1}\left(q^{n}\right)$ (see Theorem 4.4).

A second new idea is needed to deal with the contribution of matrices with $n$ small. For small $n$ the main eigenvector of $m\left(q^{n}\right)$ is not a good approximation for the contribution to the generating function. In fact, the nonprimary eigenvalues contribute to the asymptotic approximation. To overcome this difficulty we use a direct combinatorial analysis to approximate $v^{k}(n, q)$ for small $n$. This analysis appears in Section 3.

We note some similarities between this technique and the adiabatic approximation in quantum mechanics (see, for example, [15, Ch. 10]). In each case, we are sequentially applying a sequence of slowly varying matrices to a given initial vector (though in the adiabatic process, this is done continuously rather than discretely). In each case, we write our vectors in terms of the (slowly changing) eigenbasis. The final outcome is approximated by taking the product (or integral) of the eigenvalues, with a correction term due to the change of basis (known as Berry's phase in the case of quantum mechanics). The justifications for this approximation are different in the two cases, for while the adiabatic approximation holds due to cancelation of cross terms due to rapid oscillation, in our case the approximation holds because the contribution from the nonprimary eigenvectors may be safely neglected.

It should be noted that our underlying ideas have far more general applicability than simply to the problem at hand. In particular, we expect that they can be used to calculate the asymptotics of the number of partitions that locally 'avoid patterns' of various types (for example, not having any $k$ parts of consecutive sizes). For example, we believe that our techniques should be able to prove asymptotics for $p_{k, r,>B}(n)$ for all $k, r, B$. As an additional example, Knopfmacher and Munagi [19] consider the problem of counting the number of partitions $\lambda=\left(\lambda_{1}, \ldots, \lambda_{\ell}\right)$ of $n$ such that there is no $j$ with $\lambda_{j}-\lambda_{j+1}=p$ for any fixed $p>0$. The methods in this paper should also be sufficient to approximate the number of partitions of these types, although the constants showing up in the asymptotic formulas may well not have closed forms.

Bringmann, Mahlburg, and Mellit introduced a family of directed, multistate bootstrap percolation models [10]. Their study led to the following: let $\left\{E_{j}\right\}_{j=1}^{n}$ be a sequence of random variables taking values in $\{A, B, C, D\}$ such that

$$
\mathbf{P}_{s}\left(E_{j}=A\right)=\left(1-e^{-j s}\right)^{2}, \quad \mathbf{P}_{s}\left(E_{j}=B\right)=\mathbf{P}_{s}\left(E_{j}=C\right)=e^{-j s}\left(1-e^{-j s}\right),
$$




$$
\mathbf{P}_{s}\left(E_{j}=D\right)=e^{-2 j s} .
$$

They are interested in the behavior of

$$
\mathbf{P}_{s}\left(\left\{E_{j}\right\}_{j=1}^{n} \text { has no } D, C B \text {, or } C^{k}\right) \text {, }
$$

where $C^{k}$ denotes a sequence of $k$ consecutive $C$ s, as $s \downarrow 0$. Surprisingly, similar to Andrews' identity (1.2), they found a connection with a mock theta function in the case $k=2$. Again, our methods should yield an asymptotic for this probability as $s \downarrow 0$ with a relative error which is polynomial in $s^{1 / k}$.

1.3. Structure of the paper. As discussed above, our argument splits into two main pieces. On the one hand, we need a direct way of computing $v^{k}(n, q)$ for small values of $n$. Then, once we have gotten to the point where $v^{k}(n, q)$ is well approximated by the primary eigenvector of $m\left(q^{n}\right)$, we can use the recurrence relation described above.

In Section 2, we perform some preliminary calculations involving the $m\left(q^{n}\right)$ and their eigenvalues that will be used throughout. Section 3 gives a direct computation for the generating functions $v^{k}(N, q)$ for $N$ of size $s^{-1 /(k+1)-\epsilon}$. In Section 4 , we analyze the recurrence relation in order to compute $G_{k}(q)$. Section 5 contains an estimate for the product over the largest eigenvalues. Section 6 gives the proof of Theorem 1.4 and thus Theorem 1.2. Section 7 gives the proof of Theorem 1.8.

1.4. A brief note on asymptotic notation. Throughout, we will treat $k$ as constant and suppress the dependence on $k$ in our asymptotic notation. Thus, $O(X)$ will denote a quantity whose absolute value is bounded by $|X|$ times some function depending only on $k$. Similar meanings are assigned to $\Omega(X)$ and $\Theta(X)$. In particular, we will generally be interested in how these quantities behave as $s \rightarrow 0$, rather than how they behave in terms of $k$.

\section{Calculations on the diagonalization of $m\left(q^{n}\right)$}

In this section, we collect some results on the eigenvalues and diagonalization of the matrices $m\left(q^{n}\right)$. In this section, $k$ is fixed and $s$ is assumed to be small. Errors are often written in big- $O$ notation. In almost all cases the constants depend on $k$. We often suppress this dependence inside of the proofs.

Observe that the characteristic polynomial of $\left(1 / z\left(q^{n}\right)\right) m\left(q^{n}\right)$ is

$$
\lambda^{k}-z\left(q^{n}\right)^{-1}\left(\lambda^{k-1}+\cdots+\lambda+1\right) .
$$


We begin by proving some basic results about the sizes of the eigenvalues of this polynomial when $z\left(q^{n}\right)$ is either very large or very small.

REMARK 2.1. Recall that $z\left(q^{n}\right):=e^{-n s} /\left(1-e^{-n s}\right)$ is small when $n s$ is large and large when $n s$ is small.

LEMMA 2.2. For $z \in \mathbb{R}$, let $\lambda_{i}(z)$ be the roots of $\lambda^{k}-z^{-1}\left(\lambda^{k-1}+\cdots+\lambda+1\right)=0$. Then for $z$ large,

$$
\lambda_{i}(z)=\omega_{i} z^{-1 / k}\left(1+\frac{\omega_{i}}{k} z^{-1 / k}+O\left(z^{-2 / k}\right)\right)
$$

where the $\omega_{i}$ are the distinct $k$ th roots of unity. Furthermore, for $z$ small one root satisfies

$$
\lambda_{1}=z^{-1}(1+O(z))
$$

and all other roots satisfy

$$
\lambda_{i}=\omega_{i}\left(1+O_{k}(z)\right),
$$

where the $\omega_{i}$ here are distinct $k$ th roots of unity other than 1.

Proof. In order to prove the existence of roots, we will make use of Rouché theorem. In particular, we note that if there is a polynomial $q(z)=a_{0}+a_{1} z+$ $\cdots+a_{n} z^{n}$ and a positive real value $R$ so that for some $m, R^{m}\left|a_{m}\right|>\sum_{i \neq m} R^{i}\left|a_{i}\right|$, then $q$ has exactly $m$ roots (with multiplicity) in the ball of radius $R$ about 0 . We will be applying this theorem with $m=1$ and $q(z)=p\left(z+z_{0}\right)$. In particular, this implies that if $R\left|p^{\prime}\left(z_{0}\right)\right|>\left|p\left(z_{0}\right)\right|+\sum_{\ell=2}^{k} R^{\ell}\left|p^{(\ell)}\left(z_{0}\right)\right|$, then $p$ has a root within $R$ of $z_{0}$.

For the first statement, note that we only need to show this for $z \gg 1$. We claim that $p(\lambda)=\lambda^{k}-z^{-1}\left(\lambda^{k-1}+\cdots+1\right)$ has a root within $O\left(z^{-2 / k}\right)$ of $z^{-1 / k} \omega$ for every $k$ th root of unity $\omega$. This follows easily noting that $p\left(z^{-1 / k} \omega\right)=O\left(z^{-(k+1) / k}\right)$, $\left|p^{\prime}\left(z^{-1 / k} \omega\right)\right|=\Theta\left(z^{-(k-1) / k}\right)$ and that $\left|p^{(\ell)}\left(z^{-1 / k} \omega\right)\right|=O\left(z^{-(k-\ell) / k}\right)$. Applying Roche's theorem with $R$ a sufficiently large multiple of $z^{-2 / k}$ yields our result. This gives $\lambda_{i}=\omega_{i} z^{-1 / k}\left(1+O\left(z^{-1 / k}\right)\right)$. The stronger claim follows from

$$
\lambda_{i}^{k}=z^{-1}\left(1+\lambda_{i}+O\left(z^{2 / k}\right)\right) .
$$

For the later two claims, we note that it suffices to consider $z \ll 1$. For the second claim we note that $\left|p\left(z^{-1}\right)\right|=O\left(z^{-k+1}\right),\left|p^{\prime}\left(z^{-1}\right)\right|=\Theta\left(z^{-k+1}\right)$ and $\left|p^{(\ell)}\left(z^{-1}\right)\right|=O\left(z^{-k+\ell}\right)$. We then apply Roche's theorem with $R$ a sufficiently large constant. For the final claim, note that if $\omega$ is a root of $x^{k-1}+\cdots+1$ that $|p(\omega)|=O(1),\left|p^{\prime}(\omega)\right|=\Theta\left(z^{-1}\right)$ and $\left|p^{(\ell)}(\omega)\right|=O\left(z^{-1}\right)$, and apply Roche's theorem with $R$ a sufficiently large multiple of $z$. 
LEMMA 2.3. For every positive real $z$, the polynomial $p(\lambda)=\lambda^{k}-z^{-1}\left(\lambda^{k-1}+\right.$ $\cdots+\lambda+1)$ has no repeated roots.

Proof. Note that if $\lambda$ is a double root of $p$ then it is a double root of $p(x)(x-1)=$ $x^{k+1}-\left(1+z^{-1}\right) x^{k}+z^{-1}=0$ and therefore a root of the derivative of this, namely, $\left((k+1) x-\left(1+z^{-1}\right) k\right) x^{k-1}$. Since $x=0$ is clearly not a root of $p$, we have that the double root must be $x=k\left(1+z^{-1}\right) /(k+1)$. On the other hand, by Descartes Rule of Signs, $p$ has a unique, nonrepeated, real root.

Definition 2.4. By Lemma 2.3, the roots of $\lambda^{k}-z\left(q^{n}\right)^{-1}\left(\lambda^{k-1}+\cdots+\lambda+1\right)$ are distinct for any $n$ and $s$. Therefore, the eigenvalues can be analytically continued to functions of $n \in \mathbb{R}^{+}$. By Lemma 2.2, as $s \rightarrow 0$, the various eigenvalues are asymptotic to $e^{2 \pi i j / k} z^{-1 / k}$. We let $\lambda_{j}\left(q^{n}\right)$ denote the root whose analytic continuation is asymptotic to $e^{2 \pi i((j-1) / k)} z\left(q^{n}\right)^{-1 / k}$.

Thus, $\lambda_{1}\left(q^{n}\right)$ is the unique positive real root of this polynomial. We note that $\lambda_{j}\left(q^{n}\right) z\left(q^{n}\right)$ are the eigenvalues of $m\left(q^{n}\right)$ and we call $\lambda_{1}\left(q^{n}\right) z\left(q^{n}\right)$ the primary eigenvalue of the matrix $m\left(q^{n}\right)$.

REMARK 2.5. This notation differs slightly from that of Section 1.2. It is convenient for us to separate out the factor of $z\left(q^{n}\right)$ from the eigenvalue.

Since there are no repeated roots of the characteristic polynomial of $m\left(q^{n}\right)$ for each eigenvalue $z \cdot \lambda_{j}=z\left(q^{n}\right) \lambda_{j}\left(q^{n}\right)$ of $m\left(q^{n}\right)$ we have the eigenvector

$$
V_{n}^{j}:=\left(\begin{array}{c}
1 \\
\lambda_{j}^{-1} \\
\vdots \\
\lambda_{j}^{-k+1}
\end{array}\right)
$$

So we have

$$
m\left(q^{n}\right)=A\left(q^{n}\right) D\left(q^{n}\right) A\left(q^{n}\right)^{-1}
$$

with

$$
D=D\left(q^{n}\right)=\left(\begin{array}{cccc}
z \lambda_{1} & 0 & \cdots & 0 \\
0 & z \lambda_{2} & \cdots & 0 \\
& & \vdots & \\
0 & 0 & \cdots & z \lambda_{k}
\end{array}\right)
$$


and

$$
A=A\left(q^{n}\right)=\left(\begin{array}{cccc}
1 & 1 & \cdots & 1 \\
\lambda_{1}^{-1} & \lambda_{2}^{-1} & \cdots & \lambda_{k}^{-1} \\
& \vdots & \vdots & \\
\lambda_{1}^{-k+1} & \lambda_{2}^{-k+1} & \cdots & \lambda_{k}^{-k+1}
\end{array}\right)
$$

Next we turn to the transition matrices $A\left(q^{n+1}\right)^{-1} A\left(q^{n}\right)$.

LEMMA 2.6. Let $\lambda_{i}=\lambda_{i}\left(q^{n+1}\right)$ and $\mu_{i}=\lambda_{i}\left(q^{n}\right)$, then $A\left(q^{n+1}\right)=\left(\lambda_{j}^{1-i}\right)_{i, j}$ and $A\left(q^{n}\right)=\left(\mu_{j}^{1-i}\right)_{i, j}$ and

$$
\begin{aligned}
T(n, q) & =\left(T(n, q)^{i, j}\right)_{i, j}:=A\left(q^{n+1}\right)^{-1} A\left(q^{n}\right) \\
& =\left(\prod_{m \neq i}\left(\frac{\mu_{j}-\lambda_{m}}{\lambda_{i}-\lambda_{m}} \cdot \frac{\lambda_{i}}{\mu_{j}}\right)\right)_{i, j}
\end{aligned}
$$

where $i=1,2, \ldots, k$ indexes the row and $j=1,2, \ldots, k$ indexes the column of $T(n, q)$.

Proof. Note that

$$
\left(A\left(q^{n+1}\right)^{-1} A\left(q^{n}\right)\right)^{T}=A\left(q^{n}\right)^{T}\left(A\left(q^{n+1}\right)^{-1}\right)^{T} .
$$

Furthermore,

$$
A\left(q^{n}\right)^{T}\left(\begin{array}{c}
a_{0} \\
a_{1} \\
\vdots \\
a_{k-1}
\end{array}\right)=\left(\begin{array}{c}
p\left(\mu_{1}^{-1}\right) \\
p\left(\mu_{2}^{-1}\right) \\
\vdots \\
p\left(\mu_{k}^{-1}\right)
\end{array}\right)
$$

where $p(x)=a_{0}+a_{1} x+\cdots+a_{k-1} x^{k-1}$. Similarly,

$$
A\left(q^{n+1}\right)^{T}\left(\begin{array}{c}
a_{0} \\
a_{1} \\
\vdots \\
a_{k-1}
\end{array}\right)=\left(\begin{array}{c}
p\left(\lambda_{1}^{-1}\right) \\
p\left(\lambda_{2}^{-1}\right) \\
\vdots \\
p\left(\lambda_{k}^{-1}\right)
\end{array}\right)
$$

Therefore, the $(i, j)$ entry of $A\left(q^{n+1}\right)^{-1} A\left(q^{n}\right)$ is

$$
\mathbf{e}_{j}^{T} A\left(q^{n}\right)^{T}\left(A\left(q^{n+1}\right)^{-1}\right)^{T} \mathbf{e}_{i}
$$

where $\mathbf{e}_{i}$ is the vector with a 1 in the $i$ th position and zeroes in all others. This, in turn, is the value at $\mu_{j}^{-1}$ of the unique degree- $(k-1)$ polynomial $p(x)$ 
so that $p\left(\lambda_{\ell}^{-1}\right)=\delta_{\ell, i}$. Therefore,

$$
p(x)=\prod_{m \neq i} \frac{x-\lambda_{m}^{-1}}{\lambda_{i}^{-1}-\lambda_{m}^{-1}} .
$$

Thus, the $(i, j)$ entry is

$$
p\left(\mu_{j}^{-1}\right)=\prod_{m \neq i} \frac{\mu_{j}^{-1}-\lambda_{m}^{-1}}{\lambda_{i}^{-1}-\lambda_{m}^{-1}}=\prod_{m \neq i}\left(\left(\frac{\mu_{j}-\lambda_{m}}{\lambda_{i}-\lambda_{m}}\right)\left(\frac{\lambda_{i}}{\mu_{j}}\right)\right) .
$$

We will require some lemmas when dealing with transition matrices.

LEMMA 2.7. If $\lambda_{1}, \ldots, \lambda_{k}$ are the roots of $\lambda^{k}-z^{-1}\left(\lambda^{k-1}+\cdots+\lambda+1\right)=0$ and $i \neq j$, then we have

$$
\left|\lambda_{i}-\lambda_{j}\right| \gg\left|\lambda_{j}\right|
$$

Proof. By Lemma 2.2 for $|z| \gg 1$, the $\lambda_{i}$ are proportional to distinct $k$ th roots of unity, and, thus, the result follows for $z>C$ for some constant $C$.

By Lemma 2.2 for $|z| \ll 1$, all but $\lambda_{1}$, are near distinct $k$ th roots of unity, and $\lambda_{1}$ is roughly $z^{-1}$. Thus, if $i=1$ or $j=1$, then $\left|\lambda_{i}-\lambda_{j}\right| \gg z^{-1} \gg\left|\lambda_{j}\right|$. Otherwise, $\left|\lambda_{i}-\lambda_{j}\right| \gg 1 \gg\left|\lambda_{j}\right|$. Thus, the result holds for $z<c$ for some constant $c$.

For $c \leqslant z \leqslant C$, we note that $\lambda_{j} /\left(\lambda_{i}-\lambda_{j}\right)$ is a continuous function of $z$ and, thus, has some absolute upper bound. Thus, the lemma holds in this range as well.

LEMMA 2.8. In the notation of Lemma 2.6, for any $i$ and $n$ we have $\left|\mu_{i}-\lambda_{i}\right|=$ $O\left(\left|\lambda_{i}\right|\left(s+n^{-1}\right)\right)$. Moreover, we have

$$
\frac{\partial}{\partial z} \lambda_{1}(z) \ll \lambda_{1}(z)\left(1+\frac{1}{z}\right) \text { and } \frac{\partial^{2}}{\partial z^{2}} \lambda_{1}(z) \ll \lambda_{1}(z)\left(1+\frac{1}{z}\right)^{2} .
$$

Proof. The first result follows from the claim that

$$
\frac{\partial \log \left(\lambda_{i}(z)\right)}{\partial z}=O\left(z^{-1}\right)
$$

From this it follows that

$$
\begin{aligned}
\log \left(\lambda_{i} / \mu_{i}\right) & =\log \left(\lambda_{i}\left(z\left(q^{n+1}\right)\right)\right)-\log \left(\lambda_{i}\left(z\left(q^{n}\right)\right)\right) \\
& =\int_{z\left(q^{n}\right)}^{z\left(q^{n+1}\right)} \frac{\partial \log \left(\lambda_{i}(z)\right)}{\partial z} d z
\end{aligned}
$$




$$
\begin{aligned}
& =O\left(\int_{z\left(q^{n}\right)}^{z\left(q^{n+1}\right)}\left(z^{-1}\right) d z\right) \\
& =O\left(\log \left(z\left(q^{n}\right) / z\left(q^{n+1}\right)\right)\right) \\
& =O(\log (q))+O\left(\log \left(\left(1-e^{-n s}\right) /\left(1-e^{(n+1) s}\right)\right)\right) \\
& =O(s)+O\left(s /\left(1-e^{-n s}\right)\right) \\
& =O(s+1 / n) .
\end{aligned}
$$

Equation (2.5) follows from the above bounds on $\lambda_{i}$ and the identity

$$
\frac{\partial}{\partial z} \lambda_{i}(z)=-\frac{z^{-2}\left(\lambda_{i}^{k-1}+\cdots+1\right)}{k \lambda_{i}^{k-1}-z^{-1}\left((k-1) \lambda_{i}^{k-2}+\cdots+1\right)} .
$$

In particular, the above allows us to check our claim for $z \gg 1$ and for $z \ll 1$. As in Lemma 2.7, the claim follows for intermediate $z$ by a compactness argument. The bound on the second derivative follows similarly. We note that by differentiating $\lambda_{1}^{k+1}-\lambda_{1}^{k}-z^{-1}\left(\lambda_{1}^{k}-1\right)=0$ we have the identity

$$
\begin{aligned}
\left((k+1) \lambda_{1}^{k}-k \lambda_{1}^{k-1}-z^{-1} k \lambda_{1}^{k-1}\right) \frac{\partial^{2} \lambda_{1}}{\partial z^{2}} \\
=2 z^{-3}\left(\lambda_{1}^{k}-1\right)-\frac{\partial \lambda_{1}}{\partial z} \cdot 2 z^{-2} k \lambda_{1}^{k-1} \\
\quad-\left(\frac{\partial \lambda_{1}}{\partial z}\right)^{2}\left((k+1) k \lambda^{k-1}-k(k-1)\left(1+z^{-1}\right) \lambda_{1}^{k-2}\right) .
\end{aligned}
$$

LEMMA 2.9. In the notation of Lemma 2.6 for $j \neq m$

$$
\left|\frac{\mu_{i}-\lambda_{m}}{\lambda_{j}-\lambda_{m}} \cdot \frac{\lambda_{j}}{\mu_{i}}\right|
$$

is bounded by some constant depending only on $k$.

Proof. This lemma follows from Lemmas 2.7 and 2.8. In particular, in the case when $j \neq 1$ then

$$
\left|\lambda_{j}-\lambda_{m}\right| \gg\left|\lambda_{m}\right| \gg\left|\mu_{i}-\lambda_{m}\right|
$$

Thus, $\left|\left(\mu_{i}-\lambda_{m}\right) /\left(\lambda_{j}-\lambda_{m}\right)\right|$ is bounded above as is $\left|\lambda_{j} / \mu_{i}\right|$.

If $i=1$, the quantity in question is

$$
O\left(\left|\frac{\lambda_{j}}{\lambda_{j}-\lambda_{m}}\right|\right)=O(1) .
$$

Similarly, the result follows for $j=1$. 
Proposition 2.10. The transition matrix $A\left(q^{n+1}\right)^{-1} A\left(q^{n}\right)=I_{k}+O(s+1 / n)$ where $I_{k}$ is the $k \times k$ identity matrix.

Proof. We claim that

$$
T(n, q)^{i, j}=\left[A\left(q^{n+1}\right)^{-1} A\left(q^{n}\right)\right]_{i, j}=\prod_{m \neq i} \frac{\mu_{j}-\lambda_{m}}{\lambda_{i}-\lambda_{m}} \cdot \frac{\lambda_{i}}{\mu_{j}}=\delta_{i, j}+O\left(s+n^{-1}\right) .
$$

If $i \neq j$, by Lemma 2.8 the $m=j$ term of the product is

$$
\frac{\mu_{j}-\lambda_{j}}{\lambda_{i}-\lambda_{j}} \cdot \frac{\lambda_{i}}{\mu_{j}}=O\left(s+n^{-1}\right) \cdot \frac{\lambda_{i}}{\lambda_{i}-\lambda_{j}}=O\left(s+n^{-1}\right),
$$

and the remaining terms are $O(1)$ by Lemma 2.9. This proves our bound for the off-diagonal coefficients.

For $i=j$, by Lemma 2.8 each $m$-term in the above product equals

$$
\frac{\lambda_{i}-\lambda_{m}+O\left(s+n^{-1}\right)\left|\lambda_{i}\right|}{\lambda_{i}-\lambda_{m}}=1+O\left(s+n^{-1}\right),
$$

where we used that $\left|\lambda_{i}-\lambda_{m}\right| \gg\left|\lambda_{i}\right|$. Taking a product over $m$ yields $1+$ $O\left(s+n^{-1}\right)$, which proves our claim.

We conclude this section with one additional lemma dealing with the ratio of eigenvalues.

LEMMA 2.11. If $i \neq 1$ and $n s \ll 1$ then

$$
\frac{\left|\lambda_{i}\left(q^{n}\right)\right|}{\left|\lambda_{1}\left(q^{n}\right)\right|} \leqslant \exp \left(-c(n s)^{1 / k}\right)
$$

for some positive constant $c$.

Proof. This follows easily from the first case of Lemma 2.2. Namely, for $i \neq 1$

$$
\frac{\left|\lambda_{i}\right|}{\left|\lambda_{1}\right|}=\exp \left(-\Omega\left(z^{1 / k}\right)\right)=\exp \left(-\Omega\left((n s)^{1 / k}\right)\right)
$$

\section{Calculations of the early matrices}

In this section, we construct an approximation for the vector

$$
v^{k}(N, q):=\left(\widetilde{v}_{a}^{k}(N, q)\right)_{a=0}^{k-1}=\prod_{n=1}^{N} m\left(q^{n}\right) \mathbf{e}_{1}
$$


with $s^{-1 / 2} \gg N \gg s^{-1 /(k+1)} \log \left(s^{-1}\right)^{k /(k+1)}$, and $\mathbf{e}_{1}=(1,0,0, \ldots, 0)$ is the standard basis vector.

THEOREM 3.1. Assume that $k \mid N$ for some integer $N$ with $s^{-2 /(k+2)}>N$ and $N \geqslant C_{k} s^{-1 /(k+1)} \log \left(s^{-1}\right)^{k /(k+1)}$, where $C_{k}$ is a sufficiently large constant given $k$.

Then

$$
\begin{aligned}
\tilde{v}_{a}^{k}(N, q)= & (s N)^{-a / k-N((k-1) / k)} e^{N-N / k} \frac{1}{k^{3 / 2}} \\
& \times \exp \left(s^{1 / k} N^{(k+1) / k}(k+1)^{-1}+O\left(s N^{2}+s^{2 / k} N^{(k+2) / k}\right)\right) .
\end{aligned}
$$

Before proving Theorem 3.1 we introduce some notation. Each entry of the vector is the generating function for the number of partitions with no $k$-sequence, no parts larger than $N$, and the largest missing part size is $-a(\bmod k)$. In this section, we use the phrase 'run' to refer to the gap between missing parts. Given a partition $\mu$ with parts of size at most $N$ and no $k$-sequence, we let

$$
\ell=\ell(\mu)=\sum_{\text {'runs' }}(k-\text { 'length of run' }) .
$$

So for example, if all part sizes are used except for the multiples of $k$, the runs are of length $k$ and $\ell=0$. If we just miss the sizes that are $2(\bmod k)$, the first run has length 2 and the rest have length $k$, so $\ell=k-2$.

It is clear that $\ell \leqslant(k-1) N$. Note that the length of each run must be less than $k$ and that $\ell \equiv a(\bmod k)$. Let $n_{j}=n_{j}(\mu)$ be the parts not appearing in $\mu$ satisfying

$$
0<n_{1}<n_{2}<\cdots<n_{\lfloor(N+\ell) / k\rfloor}
$$

We have

$$
n_{j}=k j-\sum_{\text {'runs' before } n_{j}}(k-\text { 'length of run'). }
$$

We let $\left\{t_{j}\right\}$ be the shortenings of the runs. Namely, the length of the run before $n_{i}$ is equal to

$$
k-\left|\left\{j: t_{j}=i\right\}\right|
$$

and we have

$$
n_{i}=k i-\left|\left\{j: t_{j} \leqslant i\right\}\right| .
$$

In other words $t_{1}, t_{2}, \ldots$ is the unique weakly increasing sequence with $k-\left(n_{i}-n_{i-1}\right)$ copies of $i$ in it. So we have

$$
0 \leqslant t_{1} \leqslant t_{2} \leqslant \cdots \leqslant t_{\ell} \leqslant\left\lfloor\frac{N+\ell}{k}\right\rfloor .
$$


Note that a sequence of missing parts $\left\{n_{j}\right\}$ determines the sequence $\left\{t_{j}\right\}$ and vice versa. We set

$$
M:=\left\lfloor\frac{N+\ell}{k}\right\rfloor=\frac{N}{k}+\frac{\ell-a}{k} .
$$

Note that $n_{M}=N-a$.

So we have

$$
\widetilde{v}_{a}^{k}(N, q):=\prod_{n=1}^{N} z\left(q^{n}\right) \cdot \sum_{\ell \equiv a} \sum_{(\bmod k)} \prod_{t_{1} \leqslant \cdots \leqslant t_{\ell}} z\left(q_{i}^{n_{i}}\right)^{-1},
$$

where the sum on $\ell$ runs over $\ell \leqslant(k-1) N$. For now we ignore the term $\prod_{n=1}^{N} z\left(q^{n}\right)$ as this term can be dealt with separately. The idea for analyzing the remaining sum is that for $N$ about this size runs are likely to be of size $k$ or $k-1$. One might interpret this as saying that all the smallest parts want to appear subject to the constraint that every $k$ th part cannot appear. This agrees with Fristedt's probabilistic model of random partitions [13].

Next we give a lemma which says we can ignore large $\ell$ values.

LEMMA 3.2. In the notation above,

$$
\sum_{\substack{\ell=a \\ 2 k e N^{(k+1) / k} s^{1 / k}<\ell \leqslant(k-1) N}} \sum_{t_{1} \leqslant \cdots \leqslant t_{\ell}} \prod_{i} z\left(q^{n_{i}}\right)^{-1}=(s N)^{N / k} O\left(s^{2}\right) .
$$

Proof. We note that

$$
\begin{aligned}
\prod_{i} z\left(q^{n_{i}}\right)^{-1} & \leqslant \prod_{i} z\left(q^{N}\right)^{-1} \\
& =z\left(q^{N}\right)^{-\lfloor(N+\ell) / k\rfloor} \leqslant(s N)^{(N+\ell) / k-1} q^{O\left(N^{2}\right)} \leqslant(s N)^{N / k}(s N)^{\ell / k} s^{-1} .
\end{aligned}
$$

The number of choices for $t$ 's is $\leqslant\left(\begin{array}{c}N+\ell-1 \\ \ell\end{array}\right) \leqslant\left(\begin{array}{c}k N \\ \ell\end{array}\right)$. Thus,

$$
\sum_{t_{1} \leqslant \cdots \leqslant t_{\ell}} \prod_{i} z\left(q^{n_{i}}\right)^{-1}=O\left(s^{-1}\left(\begin{array}{c}
k N \\
\ell
\end{array}\right)(s N)^{N / k}(N s)^{\ell / k}\right) .
$$

Noting that

$$
\left(\begin{array}{c}
k N \\
\ell
\end{array}\right) \leqslant\left(\frac{k N e}{\ell}\right)^{\ell}
$$

this is at most

$$
O\left(s^{-1}(s N)^{N / k}\left(k e N^{(k+1) / k} s^{1 / k} \ell^{-1}\right)^{\ell}\right) \leqslant O\left(s^{-1}\right)(s N)^{N / k} 2^{-\ell} .
$$


We note that if $N$ is at least a sufficiently large multiple of $s^{-1 /(k+1)} \log \left(s^{-1}\right)^{k /(k+1)}$, then $2^{-\ell}=O\left(s^{3}\right)$. Summing on $\ell$, yields the result.

Proof of Theorem 3.1. We apply Lemma 3.2 to the summation in (3.2) and, unless otherwise stated, in the remainder of this proof we assume the sum on $\ell$ is truncated by $\ell<2 k e N^{(k+1) / k} s^{1 / k}$ at a cost of a negligible error. In particular, this error is at most

$$
\prod_{n=1}^{N} z\left(q^{n}\right)(s N)^{N / k} O\left(s^{2}\right),
$$

which is at most $O(s)$ times

$$
\prod_{n \leqslant N, n \neq a} z\left(q^{n}\right)
$$

which is the contribution coming from partitions with all part sizes not congruent to $a$ modulo $k$. Thus, these terms contribute an $O(s)$-fraction of the total sum (because it is at most an $O(s)$-fraction of just the terms without any parts of sizes $a(\bmod k))$, and can be safely ignored.

We will use the following calculations throughout the proof. We have $z\left(q^{n}\right)^{-1}=$ $\left(1-q^{n}\right) / q^{n}$, but $q^{n}=e^{-n s}$, so $1-q^{n}=n s(1+O(n s))$. Moreover, $\prod q^{n_{i}}=e^{-\sum n_{i} s}$ but $s \sum n_{i} \leqslant N^{2} s \ll 1$ by construction. Therefore, we have

$$
\prod_{i} z\left(q^{n_{i}}\right)^{-1}=\prod_{i} n_{i} s\left(1+O\left(n_{i}|s|\right)\right)=s^{M} \prod_{i} n_{i} \cdot\left(1+O\left(s N^{2}\right)\right) .
$$

Recall that

$$
n_{i}=k i-\left|\left\{j: t_{j} \leqslant i\right\}\right|=k i \exp \left(-\frac{\left|\left\{j: t_{j} \leqslant i\right\}\right|}{k i}+O\left(\frac{\ell\left|\left\{j: t_{j} \leqslant i\right\}\right|}{i^{2}}\right)\right) .
$$

So the sum becomes

$$
\begin{aligned}
& \sum_{\ell \equiv a} \sum_{(\bmod k)} \prod_{t_{1} \leqslant \cdots \leqslant t_{\ell}} z\left(q^{n_{i}}\right)^{-1} \\
= & \sum_{\ell \equiv a}(s k)^{M} M ! \sum_{t_{1} \leqslant \cdots \leqslant t_{\ell}} \prod_{j} \exp \left(-\sum_{i \geqslant t_{j}} \frac{1}{k i}+O\left(\frac{\ell}{i^{2}}\right)\right)\left(1+O\left(s N^{2}\right)\right) \\
= & \sum_{\ell \equiv a}(s k)^{M} \frac{M !}{\ell !} \sum_{t_{1}, \ldots, t_{\ell}} \exp \left(-\frac{1}{k} \sum_{j=1}^{\ell} \log \left(\frac{M}{t_{j}}\right)+O\left(\frac{\ell}{t_{j}}\right)\right) \\
& \times \prod_{j}\left(1+\left|\left\{i<j: t_{i}=t_{j}\right\}\right|\right)\left(1+O\left(s N^{2}\right)\right)
\end{aligned}
$$




$$
\begin{aligned}
= & \sum_{\ell \equiv a}(s k)^{M} \frac{M ! M^{\ell}}{\ell !}\left(\int_{0}^{1} t^{1 / k} e^{O(\ell / M t)} d t\right)^{\ell}\left(1+O\left(\frac{\ell^{2}}{N}+s N^{2}\right)\right) \\
= & \sum_{\ell \equiv a(\bmod k)}(s k)^{M} \frac{M ! M^{\ell}}{\ell !}\left(\int_{0}^{1} t^{1 / k}\left(1+O\left(\frac{\ell}{M t}\right)\right) d t\right)^{\ell} \\
& \times\left(1+O\left(\frac{\ell^{2}}{N}+s N^{2}\right)\right) \\
= & \sum_{\ell \equiv a}(s k)^{M} \frac{M ! M^{\ell}}{\ell !}\left(\frac{k}{k+1}\right)^{\ell}\left(1+O_{k}\left(s^{2 / k} N^{(k+2) / k}+s N^{2}\right)\right),
\end{aligned}
$$

where we use that $\delta \leqslant((k+1) / k) \epsilon$. The third line is obtained by removing the ordering on the $t_{i}$ 's. The product $(1 / \ell !) \prod_{j}\left(1+\left|\left\{i<j: t_{i}=t_{j}\right\}\right|\right)$ accounts for the introduced overcounting. The fourth line is obtained by approximating the sum over $t_{j}$ (once $t_{i}$ has been fixed for $i<j$ ) of $t_{j}^{1 / k}\left(1+\left|\left\{i<j: t_{i}=t_{j}\right\}\right|\right.$ ) by $M\left(\int t^{1 / k} d t\right)(1+O(\ell / N))$. Additionally, in the fifth line we note that term $O(\ell / M t)$ is always negative, see (3.3).

Applying Stirling's approximation to $M$ !, and suppressing the errors, we see that the above is equal to

$$
\begin{aligned}
\left(\frac{s}{e}(N-a)\right)^{(N-a) / k} \sqrt{2 \pi \frac{N-a}{k}} \sum_{\ell \equiv a(\bmod k)}\left(\frac{s}{e}\right)^{\ell / k}\left(\frac{N+\ell-a}{N-a}\right)^{(N-a) / k+1 / 2} \\
\quad \times(N+\ell-a)^{\ell((k+1) / k)} \frac{1}{\ell !}\left(\frac{1}{k+1}\right)^{\ell} \\
=\left(\frac{s(N-a)}{e}\right)^{(N-a) / k} \sqrt{2 \pi \frac{N-a}{k}} \\
\quad \times \sum_{\ell \equiv a(\bmod k)}\left(\frac{1}{k+1} s^{1 / k}(N-a)^{(k+1) / k}\left(1+O\left(\frac{\ell}{N}\right)\right)\right)^{\ell} \frac{1}{\ell !} \\
=\left(\frac{s(N-a)}{e}\right)^{(N-a) / k} \sqrt{2 \pi \frac{N-a}{k}} \\
\quad \times\left(\sum_{\ell \equiv a(\bmod k)}\left(\frac{1}{k+1} s^{1 / k}(N-a)^{(k+1) / k}\right)^{\ell} \frac{1}{\ell !}\right)\left(1+O\left(s^{2 / k} N^{(k+2) / k}\right)\right)
\end{aligned}
$$

where we have used $((N+\ell-a) /(N-a))^{(N-a) / k}=(1+\ell / N)^{(N-a) / k}=e^{\ell / k}$ times a negligible error.

Extending the sum to a sum over all $\ell$ rather than those with $\ell<2 k e s^{1 / k} N^{(k+1) / k}$ introduces a negligible error. The completed sum over $\ell$ is the sum over every 
$k$ th term of an exponential. Thus, letting $\omega$ be a primitive $k$ th root of unity suppressing the above error terms, we have

$$
\begin{aligned}
& \sum_{\ell \equiv a}\left(s_{(\bmod k)}(N-a)^{(k+1) / k}(k+1)^{-1}\right)^{\ell} \frac{1}{\ell !} \\
= & \frac{1}{k} \sum_{t} \omega_{(\bmod k)}^{a t} \exp \left(s^{1 / k}(N-a)^{(k+1) / k}(k+1)^{-1} \omega^{-t}\right) \\
= & \frac{1}{k} \exp \left(s^{1 / k}(N-a)^{(k+1) / k}(k+1)^{-1}\right) \\
& \times\left(1+O\left(\exp \left(-\Omega\left(\frac{s^{1 / k} N^{(k+1) / k}}{k^{2}(k+1)}\right)\right)\right)\right) \\
= & \frac{1}{k} \exp \left(s^{1 / k} N^{(k+1) / k}(k+1)^{-1}\right)\left(1+O\left((s N)^{1 / k}\right)\right)
\end{aligned}
$$

where we have approximated $N-a$ by $N$, and noted that the $t \neq 0$ terms are smaller by a factor of $\exp \left(-\Omega\left(s^{1 / k} N^{(k+1) / k}\right)\right)$, which is $O\left(s^{3}\right)$ for $N$ a sufficiently large multiple of $s^{-1 /(k+1)} \log \left(s^{-1}\right)^{k /(k+1)}$.

To finish the proof of the theorem we use

$$
\begin{aligned}
\prod_{n=1}^{N} z\left(q^{n}\right) & =\prod_{n=1}^{N}(s n)^{-1}(1+O(n s))=\frac{s^{-N}}{N !}\left(1+O\left(N^{2} s\right)\right) \\
& =\frac{e^{N}}{(s N)^{N} \sqrt{2 \pi N}}\left(1+O\left(N^{2} s\right)\right) .
\end{aligned}
$$

Before concluding this section we give a comparison between $\widetilde{v}_{0}^{k}(N, q)$ and the eigenvectors of $m\left(q^{N}\right)$. We let $V_{n}^{i}(q)$ be the eigenvector

$$
\left(\begin{array}{llll}
1 & \lambda_{i}\left(q^{n}\right)^{-1} & \cdots & \left.\lambda_{i}\left(q^{n}\right)^{-k+1}\right)^{T}
\end{array}\right.
$$

of $m\left(q^{n}\right)$ corresponding to the eigenvalue $\lambda_{i}\left(q^{n}\right) z\left(q^{n}\right)$.

PROPOSITION 3.3. In the notation above, with the assumptions of Theorem 3.1 and

$$
V_{N}^{i}(q)=\left(\begin{array}{llll}
1 & \lambda_{i}\left(q^{N}\right)^{-1} & \ldots & \lambda_{i}\left(q^{N}\right)^{-k+1}
\end{array}\right)^{T}
$$

we have

$$
\begin{aligned}
v^{k}(N, q)= & (N s)^{-N((k-1) / k)} e^{N-N / k} \frac{1}{k^{3 / 2}} \exp \left(s^{1 / k} N^{(k+1) / k}(k+1)^{-1}\right. \\
& \left.+O\left(s N^{2}+s^{2 / k} N^{(k+2) / k}\right)\right) V_{N}^{1}(q)+\sum_{i>1} C_{N}^{i}(q) V_{N}^{i}(q)
\end{aligned}
$$


where

$C_{N}^{i}(q) \ll(N s)^{-N((k-1) / k)} e^{N-N / k} \exp \left(s^{1 / k} N^{(k+1) / k}(k+1)^{-1}\right) O\left(s N^{2}+s^{2 / k} N^{(k+2) / k}\right)$.

Proof. Since the eigenvectors form a basis, there exist $C_{N}^{i}(q)$ so that $v^{k}(N, q)=$ $\sum_{i \geqslant 1} C_{N}^{i}(q) V_{N}^{i}(q)$. Applying Theorem 3.1, we have that

$$
\widetilde{v}_{a}^{k}(N, q)=\widetilde{v}_{0}^{k}(N, q)(s N)^{-a / k}\left(1+O\left(s^{2 / k} N^{(k+2) / k}+s N^{2}\right)\right)
$$

By Lemma 2.2 we have that

$$
\lambda_{j}\left(q^{N}\right)=e^{2 \pi i((j-1) / k)}(s N)^{1 / k}\left(1+O\left((s N)^{2 / k}\right)\right)
$$

Therefore, we have that for $0 \leqslant a \leqslant k-1$,

$\widetilde{v}_{0}^{k}(N, q)\left(1+O\left(s^{2 / k} N^{(k+2) / k}+s N^{2}\right)\right)=\sum_{j=1}^{k} e^{-2 \pi i a(j-1) / k}\left(1+O(s N)^{2 / k}\right) C_{N}^{j}(q)$.

In other words if $B$ is the matrix with $(a, j)$ entry $e^{-2 \pi i a(j-1) / k}$ (with $a$ running 0 to $k-1$ and $j$ running 1 to $k)$, then $B+O(s N)^{2 / k}$ times the vector of $C_{N}^{i}(q)$ equals a vector whose entries are $\widetilde{v}_{0}^{k}(N, q)\left(1+O\left(s^{2 / k} N^{(k+2) / k}+s N^{2}\right)\right)$. Noting that the inverse of $B+O(s N)^{2 / k}$ is $B^{-1}+O(s N)^{2 / k}$ this implies that

$$
C_{N}^{1}(q)=\widetilde{v}_{0}^{k}(N)\left(1+O\left(s^{2 / k} N^{(k+2) / k}+s N^{2}\right)\right)
$$

and $C_{N}^{i}(q)=\widetilde{v}_{0}^{k}(N, q) O\left(s^{2 / k} N^{(k+2) / k}+s N^{2}\right)$ for $i>1$. This proves our proposition.

Finally, the next proposition compares $\widetilde{v}_{0}^{k}(N, q)$ to the product of the eigenvalues.

PROPOSITION 3.4. In the notation above, with the assumptions of Theorem 3.1 we have

$$
\begin{aligned}
\frac{\widetilde{v}_{0}^{k}(N, q)}{\prod_{n=1}^{N} \lambda_{1}\left(q^{n}\right) z\left(q^{n}\right)}= & \frac{1}{k^{3 / 2}(2 \pi)^{(1-k) / 2 k}} \\
& \times \exp \left(\frac{k-1}{2 k} \log (N)+O\left(s^{2 / k} N^{(k+2) / k}+s N^{2}\right)\right) .
\end{aligned}
$$


Proof. By Lemma 2.2 we see that the product of the first $N$ primary eigenvalues is

$$
\begin{aligned}
\prod_{n=1}^{N} \lambda_{1}\left(q^{n}\right) z\left(q^{n}\right)= & \prod_{n=1}^{N}(n s)^{1 / k}\left(1+\frac{1}{k}(n s)^{1 / k}+O(n s)^{2 / k}\right) \cdot(n s)^{-1}(1+O(n s)) \\
= & \prod_{n=1}^{N}(n s)^{-(k-1) / k}\left(1+\frac{1}{k}(n s)^{1 / k}+O(n s)^{2 / k}\right) \\
= & (N !)^{-(k-1) / k} s^{-((k-1) / k) N} \exp \left(\frac{s^{1 / k}}{k+1} N^{(1+k) / k}+O\left((s N)^{1 / k}\right)\right) \\
= & (2 \pi)^{-(k-1) / 2 k}(N s)^{-N((k-1) / k)} e^{N(1-1 / k)} \\
& \times \exp \left(-\frac{k-1}{2 k} \log (N)+\frac{1}{k+1} s^{1 / k} N^{(k+1) / k}+O\left((s N)^{1 / k}\right)\right) .
\end{aligned}
$$

Theorem 3.1 gives the result.

\section{After the run-up}

In the previous section, we computed $\widetilde{V}(N, q)=\prod_{n=1}^{N} m\left(q^{n}\right) \mathbf{e}_{1}$. In this section, we evaluate

$$
G_{k}(q)=\mathbf{e}_{1}^{T} \prod_{n=N}^{\infty} m\left(q^{n}\right) \widetilde{V}(N, q) .
$$

We have the following proposition which shows that we only need to consider the eigenvalues and the first entry in each of the transition matrices.

THEOREM 4.1. In the notation from Lemma 2.6 for $N$ an integer bigger than a sufficiently large multiple of $s^{-1 /(k+1)} \log \left(s^{-1}\right)^{k /(k+1)}$ we have

$$
\begin{aligned}
G_{k}(q)= & \prod_{n=N}^{\infty} \lambda_{1}\left(q^{n}\right) z\left(q^{n}\right) \cdot \prod_{n=N}^{\infty} T(n, q)^{1,1} \\
& \cdot \widetilde{v}_{0}^{k}(N, q) \cdot\left(1+O\left(s+N^{(-k-1) / k} s^{-1 / k}\right)\right) .
\end{aligned}
$$

In order to prove Theorem 4.1 we will need the following lemma.

LemMA 4.2. Let $w(n, q):=A\left(q^{n}\right)^{-1} \prod_{i=1}^{n-1} m\left(q^{i}\right) \mathbf{e}_{1}$. Then for $n$ bigger than a sufficiently large multiple of $s^{-1 /(k+1)} \log \left(s^{-1}\right)^{k /(k+1)}$, we have that for $i \neq 1$ that

$$
\left|w(n, q)_{i}\right| \leqslant O\left(n^{-(k+1) / k} s^{-1 / k}+s\right)\left|w(n, q)_{1}\right| .
$$


Proof of Lemma 4.2. The proof is by induction on $n$. Proposition 3.3 makes this result clear for $n$ at the lowest end of the permissible range. The basic idea here is that

$$
w(n+1, q)=T(n, q) D\left(q^{n}\right) w(n, q) .
$$

Now since $\left|\lambda_{1}\left(q^{n}\right)\right|>\left|\lambda_{i}\left(q^{n}\right)\right|$, multiplication by $D\left(q^{n}\right)$ increases the ratio of the first entry relative to the other entries. Since $T(n, q)$ is approximately $I$, multiplication by $T(n, q)$ does not worsen this ratio by too much.

We begin by proving our claim for $n s \ll 1$. Letting

$$
u(n, q):=D\left(q^{n}\right) w(n, q)
$$

and applying Lemma 2.2, we have that

$$
\frac{\left|u(n, q)_{i}\right|}{\left|u(n, q)_{1}\right|}=\frac{\left|w(n, q)_{i}\right|\left|\lambda_{i}\left(q^{n}\right)\right|}{\left|w(n, q)_{1}\right|\left|\lambda_{1}\left(q^{n}\right)\right|} \leqslant \frac{\left|w(n, q)_{i}\right|}{\left|w(n, q)_{1}\right|}\left(1-\Omega\left((n s)^{1 / k}\right)\right) .
$$

Next, since $T(n, q)=I_{k}+O\left(n^{-1}\right)$, we have that

$$
\frac{\left|w(n+1, q)_{i}\right|}{\left|w(n+1, q)_{1}\right|}=O\left(n^{-1}\right)+\left(\frac{\left|w(n, q)_{i}\right|}{\left|w(n, q)_{1}\right|}\right)\left(1-\Omega\left((n s)^{1 / k}\right)\right) .
$$

Induction on $n$ gives

$$
\left|w(n, q)_{i}\right| \leqslant O\left(n^{-(k+1) / k} s^{-1 / k}\right)\left|w(n, q)_{1}\right|
$$

for all $n \ll s^{-1}$.

The argument for $n s \gg 1$ is similar. It should be noted that in this range that $\left|\lambda_{i}\left(q^{n}\right)\right| /\left|\lambda_{1}\left(q^{n}\right)\right|$ is bounded above by some constant less than 1 (say by $1-\epsilon$ ). Therefore, we have that

$$
\frac{\left|w(n+1, q)_{i}\right|}{\left|w(n+1, q)_{1}\right|}=O(s)+\left(\frac{\left|w(n, q)_{i}\right|}{\left|w(n, q)_{1}\right|}\right)(1-\epsilon) .
$$

From this, it is easy to conclude by induction that $\left|w(n, q)_{i}\right|=O(s)\left|w(n, q)_{1}\right|$.

REMARK 4.3. It should be noted that the bound in Lemma 4.2 is not tight for small $n$ (a stronger bound is given in Proposition 3.3). The bound of $n^{-(k+1) / k} s^{-1 / k}$ would be tight given our analysis if all we use is that $T(n$, $q)^{1, i}=O\left(n^{-1}\right)$ and that $\left|\lambda_{i}\left(q^{n}\right) / \lambda_{1}\left(q^{n}\right)\right|=1-\Omega\left((n s)^{1 / k}\right)$. In order to obtain a tighter analysis, one can note that the $T(n, q)^{1, i}$ are roughly constant in $n$ and that $\lambda_{i} / \lambda_{1}$ is roughly $\omega^{i}$, where $\omega$ is a primitive $k$ th root of unity. By our previous 
analysis, $w_{i}(n+1, q) / w_{1}(n+1, q)$ is approximately $\left(\lambda_{i}\left(q^{n}\right) / \lambda_{1}\left(q^{n}\right)\right)(T(n$, $\left.q)^{1, i}+\left(w_{i}(n, q) / w_{1}(n, q)\right)\right)$. Approximating each $\lambda_{i} / \lambda_{1}$ by $\omega^{i}\left(1-(n s)^{1 / k}\right)$ and each $T(n, q)^{1, i}$ by a constant of order $n^{-1}$, we note that the resulting recurrence leads to terms of size $O\left(n^{-1}\right)$ due to cancelation that is not captured in our analysis.

We are now prepared to prove Theorem 4.1.

Proof of Theorem 4.1. We claim that

$$
\begin{aligned}
w(n+1, q)_{1}= & w(n, q)_{1} \lambda_{1}\left(q^{n}\right) z\left(q^{n}\right) T(n, q)^{1,1} \\
& \times\left(1+O\left(\min \left(n^{-(2 k+1) / k} s^{-1 / k}, s^{2} z\left(q^{n}\right)\right)\right)\right) .
\end{aligned}
$$

Or equivalently (since $\left.u(n, q)_{1}=\lambda_{1}\left(q^{n}\right) z\left(q^{n}\right) w(n, q)_{1}\right)$ that

$$
w(n+1, q)_{1}=u(n, q)_{1} T(n, q)^{1,1}\left(1+O\left(\min \left(n^{-(2 k+1) / k} s^{-1 / k}, s^{2} z\left(q^{n}\right)\right)\right)\right) .
$$

It is clear that

$$
w(n+1, q)_{1}=\sum_{j} T(n, q)^{1, j} u(n, q)_{j} .
$$

Hence, we need to show

$$
\max _{j \neq 1}\left(T(n, q)^{1, j} \cdot \frac{\left|u(n, q)_{j}\right|}{\left|u(n, q)_{1}\right|}\right)=O\left(\min \left(n^{-(2 k+1) / k} s^{-1 / k}+s, s^{2} z\left(q^{n}\right)\right)\right) .
$$

If $n s \ll 1$, this follows since $T(n, q)^{1, j} \ll n^{-1}$ by Proposition 2.10, and $\left|u(n, q)_{j}\right| /\left|u(n, q)_{1}\right| \leqslant\left|w(n, q)_{j}\right| /\left|w(n, q)_{1}\right|=O\left(n^{-(k+1) / k} s^{-1}\right)$ by Lemma 4.2. Otherwise, this follows from noting that $T(n, q)^{1, j} \ll s$ by Proposition 2.10 and

$$
\frac{\left|u(n, q)_{j}\right|}{\left|u(n, q)_{1}\right|}=\left(\frac{\left|\lambda_{j}\left(q^{n}\right)\right|}{\left|\lambda_{1}\left(q^{n}\right)\right|}\right)\left(\frac{\left|w(n, q)_{j}\right|}{\left|w(n, q)_{1}\right|}\right)=O\left(z\left(q^{n}\right) s\right)
$$

by Lemma 2.2. This proves the claim.

Therefore, we have that

$$
\begin{aligned}
\lim _{n \rightarrow \infty} w(n, q)_{1}= & w(N, q)_{1} \prod_{n=N+1}^{\infty} \lambda_{1}\left(q^{n}\right) z\left(q^{n}\right) T(n, q)^{1,1} \\
& \cdot \exp \left(O\left(\sum_{n=N+1}^{\infty} \min \left(n^{-(2 k+1) / k} s^{-1 / k}, s^{2} z\left(q^{n}\right)\right)\right)\right) .
\end{aligned}
$$

The sum in the error term is at most

$$
\sum_{n=N+1}^{\left\lfloor s^{-1}\right\rfloor} n^{-(2 k+1) / k} s^{-1 / k}+\sum_{n=\left\lfloor s^{-1}\right\rfloor}^{\infty} s^{2} z\left(q^{n}\right) .
$$


The first term is $O\left(N^{-(k+1) / k} s^{-1 / k}\right)$ and the latter term is $O\left(s^{2} \sum_{n=1}^{\infty} e^{-n s}\right)=$ $O(s)$. Combining with Proposition 3.3 yields our result.

The following theorem is enough to deduce Theorem 1.4 and, thus, Theorem 1.2.

THEOREM 4.4. With $N$ as above we have

$$
\prod_{n=N}^{\infty} T(n, q)^{1,1}=k^{1 / 2} \exp \left(-\frac{k-1}{2 k} \log (N s)+O\left((N s)^{1 / k}+N^{-1}+s\right)\right) .
$$

Proof. Throughout this proof we use the notation of Lemma 2.6 and often suppress the dependence on $n$. We have

$$
T(n-1, q)^{1,1}=\prod_{m \neq 1} \frac{\mu_{1}-\lambda_{m}}{\lambda_{1}-\lambda_{m}} \cdot \frac{\lambda_{1}}{\mu_{1}}
$$

and

$$
\mu_{1}\left(q^{n}\right)=\lambda_{1}\left(q^{n-1}\right)=\lambda_{1}\left(q^{n}\right)-\lambda_{1}^{\prime}\left(q^{n}\right)+O\left(\lambda_{1}^{\prime \prime}\left(q^{n}\right)\right)
$$

where $\lambda_{1}^{\prime}\left(q^{n}\right)=(\partial / \partial n) \lambda_{1}\left(q^{n}\right)$. Therefore,

$$
\frac{\mu_{1}-\lambda_{m}}{\lambda_{1}-\lambda_{m}} \cdot \frac{\lambda_{1}}{\mu_{1}}=1-\lambda_{1}^{\prime}\left(\frac{1}{\lambda_{1}-\lambda_{m}}-\frac{1}{\lambda_{1}}\right)+O\left(\left(\frac{\lambda_{1}^{\prime \prime}}{\lambda_{1}}+\left(\frac{\lambda_{1}^{\prime}}{\lambda_{1}}\right)^{2}\right)\right) .
$$

Hence,

$$
T(n-1, q)^{1,1}=\exp \left(-\lambda_{1}^{\prime} \sum_{m \neq 1}\left(\frac{1}{\lambda_{1}-\lambda_{m}}-\frac{1}{\lambda_{1}}\right)+O\left(\left(\frac{\lambda_{1}^{\prime \prime}}{\lambda_{1}}+\left(\frac{\lambda_{1}^{\prime}}{\lambda_{1}}\right)^{2}\right)\right)\right) .
$$

To estimate the big- $O$ term for $n s \ll 1$ we use (2.6) and (2.7) and Lemma 2.2 to obtain

$$
\begin{aligned}
\frac{1}{\lambda_{1}} \frac{\partial \lambda_{1}}{\partial n} & =-s \frac{1}{\lambda_{1}} \frac{\partial \lambda_{1}}{\partial z} \cdot z\left(q^{n}\right)^{2} e^{n s}=O\left(\frac{1}{n}\right) \\
\frac{1}{\lambda_{1}} \frac{\partial^{2} \lambda_{1}}{\partial n^{2}} & =\frac{s^{2} e^{2 n s}}{\lambda_{1}}\left(\frac{\partial^{2} \lambda_{1}}{\partial z^{2}} \cdot z\left(q^{n}\right)^{4}+\frac{\partial \lambda_{1}}{\partial z} \cdot z\left(q^{n}\right)^{3}\right)=O\left(\frac{1}{n^{2}}\right) .
\end{aligned}
$$

For $n s \gg 1$ we use Lemma 2.8 to obtain

$$
\frac{1}{\lambda_{1}} \frac{\partial \lambda_{1}}{\partial n}=O(s) \text { and } \quad \frac{1}{\lambda_{1}} \frac{\partial^{2} \lambda_{1}}{\partial n^{2}}=O\left(s^{2}\right)
$$


Therefore,

$$
\begin{aligned}
& \prod_{n=N}^{\left\lfloor s^{-3 / 2}\right\rfloor} T(n-1, q)^{1,1} \exp \left(\lambda_{1}^{\prime} \sum_{m \neq 1}\left(\frac{1}{\lambda_{1}-\lambda_{m}}-\frac{1}{\lambda_{1}}\right)\right) \\
& \quad=\exp \left(\sum_{n=N}^{\lfloor 1 / s\rfloor} O\left(\frac{1}{n^{2}}\right)+O\left(\sum_{n=\lfloor 1 / s\rfloor}^{\left\lfloor s^{-3 / 2}\right\rfloor} s^{2}\right)\right) \\
& \quad=\exp \left(O\left(\frac{1}{N}+s^{1 / 2}\right)\right) .
\end{aligned}
$$

On the other hand for $n \geqslant s^{-3 / 2}$ (which implies $n s \gg 1$ ), it is easy to see that both $\log \left(T(n, q)^{1,1}\right)$ and $\lambda_{1}^{\prime} \sum_{m \neq 1}\left(1 /\left(\lambda_{1}-\lambda_{m}\right)-1 / \lambda_{1}\right)$ are $O(z)=O\left(e^{-n s}\right)$. And therefore,

$$
\prod_{n=\left\lfloor s^{-3 / 2}\right\rfloor}^{\infty} T(n-1, q)^{1,1} \exp \left(\lambda_{1}^{\prime} \sum_{m \neq 1}\left(\frac{1}{\lambda_{1}-\lambda_{m}}-\frac{1}{\lambda_{1}}\right)\right)=\exp \left(O\left(e^{s^{-1 / 2}} / s\right)\right) .
$$

Let $P(\lambda, z):=\lambda^{k}-z^{-1}\left(\lambda^{k-1}+\cdots+\lambda+1\right)$. We have

$$
2 \sum_{m \neq 1} \frac{1}{\lambda_{1}-\lambda_{m}}=\left.\frac{\left(\partial^{2} / \partial \lambda^{2}\right) P(\lambda, z)}{(\partial / \partial \lambda) P(\lambda, z)}\right|_{\substack{\lambda=\lambda_{1} \\ z=z(N, q)}}=: R_{k}\left(\lambda_{1}\left(q^{n}\right)\right)
$$

Therefore,

$$
\begin{aligned}
\prod_{n=N}^{\left\lfloor s^{-3 / 2}\right\rfloor} T(n, q)^{1,1}= & \prod_{n=N}^{\left\lfloor s^{-3 / 2}\right\rfloor} T(n-1, q)^{1,1}\left(1+O\left(N^{-1}+s\right)\right) \\
= & \exp \left(-\sum_{n=N}^{\left\lfloor s^{-3 / 2}\right\rfloor}\left(\frac{1}{2} \lambda_{1}^{\prime}\left(q^{n}\right) R_{k}\left(\lambda_{1}\left(q^{n}\right)\right)-(k-1) \frac{\lambda_{1}^{\prime}\left(q^{n}\right)}{\lambda_{1}\left(q^{n}\right)}\right)\right. \\
& \left.+O\left(N^{-1}+s^{1 / 2}\right)\right) .
\end{aligned}
$$

We apply Euler-MacLaurin to approximate the sum by an integral. The error from the terms $\lambda_{1}^{\prime}\left(q^{n}\right) / \lambda_{1}\left(q^{n}\right)$ introduces an error of size

$$
\int_{N}^{\left\lfloor s^{-3 / 2}\right\rfloor}\left(\frac{\lambda_{1}^{\prime \prime}\left(q^{n}\right)}{\lambda_{1}\left(q^{n}\right)}+\left(\frac{\lambda_{1}^{\prime}\left(q^{n}\right)}{\lambda_{1}\left(q^{n}\right)}\right)^{2}\right) d n=O\left(N^{-1}+s^{1 / 2}\right)
$$


as above. Thus, we have

$$
\begin{aligned}
\prod_{n=N}^{\left\lfloor s^{-3 / 2}\right\rfloor} T(n, q)^{1,1}= & \exp \left(-\int_{N}^{\left\lfloor s^{-3 / 2}\right\rfloor}\left(\frac{1}{2} \lambda_{1}^{\prime}\left(q^{x}\right) R_{k}\left(\lambda_{1}\left(q^{x}\right)\right)-(k-1) \frac{\lambda_{1}^{\prime}\left(q^{x}\right)}{\lambda_{1}\left(q^{x}\right)}\right) d x\right. \\
& \left.+O\left(N^{-1}+s^{1 / 2}\right)\right) .
\end{aligned}
$$

Note that since the terms in the product are exponentially close to 1 for larger $n$ and the terms in the integral are exponentially small, we get that

$$
\begin{aligned}
\prod_{n=N}^{\infty} T(n, q)^{1,1}= & \exp \left(-\int_{N}^{\infty}\left(\frac{1}{2} \lambda_{1}^{\prime}\left(q^{x}\right) R_{k}\left(\lambda_{1}\left(q^{x}\right)\right)-(k-1) \frac{\lambda_{1}^{\prime}\left(q^{x}\right)}{\lambda_{1}\left(q^{x}\right)}\right) d x\right. \\
& \left.+O\left(N^{-1}+s^{1 / 2}\right)\right) \\
= & \exp \left(-\int_{\lambda_{1}\left(q^{N}\right)}^{\infty} \frac{R_{k}(x)}{2}-\frac{k-1}{x} d x+O\left(N^{-1}+s^{1 / 2}\right)\right) .
\end{aligned}
$$

In order to evaluate the integral $\int R_{k}(x) d x$, we let $a(\lambda)=\lambda^{k}$ and $b(\lambda)=$ $\lambda^{k-1}+\cdots+1$. We then have that $z^{-1}=a\left(\lambda_{1}\right) / b\left(\lambda_{1}\right)$. Therefore,

$$
\begin{aligned}
R_{k}(\lambda) & =\frac{a^{\prime \prime}(\lambda)-z^{-1} b^{\prime \prime}(\lambda)}{a^{\prime}(\lambda)-z^{-1} b^{\prime}(\lambda)} \\
& =\frac{a^{\prime \prime}(\lambda) b(\lambda)-a(\lambda) b^{\prime \prime}(\lambda)}{a^{\prime}(\lambda) b(\lambda)-a(\lambda) b^{\prime}(\lambda)}=\frac{\partial}{\partial \lambda} \log \left(a^{\prime}(\lambda) b(\lambda)-a(\lambda) b^{\prime}(\lambda)\right) .
\end{aligned}
$$

Letting

$$
\begin{aligned}
Q(\lambda) & :=a^{\prime}(\lambda) b(\lambda)-a(\lambda) b^{\prime}(\lambda) \\
& =k \lambda^{k-1}\left(\lambda^{k-1}+\cdots+1\right)-\lambda^{k}\left((k-1) \lambda^{k-2}+\cdots+1\right) \\
& =\lambda^{2 k-2}+2 \lambda^{2 k-3}+\cdots+k \lambda^{k-1},
\end{aligned}
$$

we have that

$$
\int_{\lambda_{1}\left(q^{N}\right)}^{\infty} \frac{R_{k}(x)}{2}-\frac{k-1}{x} d x=\frac{1}{2}\left[\log \left(Q(\lambda) \lambda^{-2 k+2}\right)\right]_{\lambda_{1}(N)}^{\infty} .
$$

We note that for $\lambda \gg 1$ that $Q(\lambda) \lambda^{-2 k+2}=1+O\left(\lambda^{-1}\right)$, and therefore,

$$
\lim _{\lambda \rightarrow \infty} \log \left(Q(\lambda) \lambda^{-2 k+2}\right)=0 .
$$

For $\lambda \ll 1$, we have that $Q(\lambda) \lambda^{-2 k+2}=k \lambda^{-k+1}(1+O(\lambda))$. Therefore,

$$
\prod_{n=N}^{\infty} T(n, q)^{1,1}=\exp \left(\frac{1}{2} \log \left(k \lambda_{1}\left(q^{N}\right)^{-k+1}\left(1+O\left(\lambda_{1}\left(q^{N}\right)\right)\right)\right)+O\left(N^{-1}+s^{1 / 2}\right)\right) \text {. }
$$


By Lemma 2.2, we have

$$
\begin{aligned}
\prod_{n=N}^{\infty} T(n, q)^{1,1}= & \exp \left(-\frac{k-1}{2} \log \left(\lambda_{1}\left(q^{N}\right)\right)+\frac{1}{2} \log (k)\right. \\
& \left.+O\left((N s)^{1 / k}+N^{-1}+s\right)\right) \\
= & \exp \left(-\frac{k-1}{2 k} \log (N s)+\frac{1}{2} \log (k)\right. \\
& \left.-\frac{k-1}{2 k}(N s)^{1 / k}+O\left((N s)^{1 / k}+N^{-1}+s\right)\right) .
\end{aligned}
$$

In the next section, we analyze the product of the primary eigenvalues.

\section{The product of the primary eigenvalues}

In this section, we estimate

$$
\prod_{n=1}^{\infty} \lambda_{1}\left(q^{n}\right) z\left(q^{n}\right)=\exp \left(\sum_{n=1}^{\infty} \log \left(\lambda_{1}\left(q^{n}\right)\right)+\log \left(\frac{q^{n}}{1-q^{n}}\right)\right) .
$$

THEOREM 5.1. In the notation above we have

$$
\begin{aligned}
\sum_{n=1}^{\infty} \log \left(\lambda_{1}\left(q^{n}\right) z\left(q^{n}\right)\right)= & \frac{\pi^{2}}{6 s}\left(1-\frac{2}{k(k+1)}\right)+\left(\frac{k-1}{2 k}\right) \log (s) \\
& -\left(\frac{k-1}{2 k}\right) \log (2 \pi)+O_{k}\left(s^{1 / k}\right) .
\end{aligned}
$$

We start with the following lemma which closely resembles Euler-MacLaurin summation.

LEMMA 5.2. For suitable functions $h$ and $n \geqslant 1$ we have

$$
\begin{aligned}
h(n) & =\int_{n-1 / 2}^{n+1 / 2} h(z) d z-\int_{n-1 / 2}^{n+1 / 2} h^{\prime}(x)\left([x]-x+\frac{1}{2}\right) d x \\
& =\int_{n-1 / 2}^{n+1 / 2} h(z) d z-\frac{1}{2} \int_{n-1 / 2}^{n+1 / 2} h^{\prime \prime}(x)\left([x]-x+\frac{1}{2}\right)^{2} d x
\end{aligned}
$$

where $[x]$ denotes the integer part of $x$. 
Proof. To see this note that for any function $h(z)$ we have

$$
h(z)=h(n)+h^{\prime}(n)(z-n)+\int_{n}^{z} h^{\prime \prime}(x)(z-x) d x .
$$

Integrating from $n-1 / 2$ to $n+1 / 2$ gives the second result. Integration by parts on each interval $[n, n+1 / 2]$ and $[n-1 / 2, n]$ gives the first result.

Define the function $f_{k}\left(e^{-x}\right)$ to be the increasing function satisfying

$$
f_{k}\left(e^{-x}\right)^{k+1}-f_{k}\left(e^{-x}\right)^{k}=e^{-x(k+1)}-e^{-x k} .
$$

Since $\lambda_{1}\left(q^{n}\right)^{k}=z\left(q^{n}\right)^{-1}\left(\lambda_{1}\left(q^{n}\right)^{k-1}+\cdots \lambda_{1}\left(q^{n}\right)+1\right)$, multiplying by $\lambda_{1}\left(q^{n}\right)-1$ we have $\lambda_{1}\left(q^{n}\right)^{k+1}-\lambda_{1}\left(q^{n}\right)^{k}=z\left(q^{n}\right)^{-1}\left(\lambda_{1}\left(q^{n}\right)^{k}-1\right)=q^{-n} \lambda_{1}^{k}-q^{-n}-\lambda_{1}^{k}+1$. Therefore $f_{k}\left(e^{-n s}\right)=\lambda_{1}\left(q^{n}\right) q^{n}$.

REMARK 5.3. This function $f_{k}\left(e^{-x}\right)$, and certain generalizations, are studied in [17].

Proof of Theorem 5.1. The modularity of the Dedekind $\eta$-function gives

$$
\sum_{n=1}^{\infty} \log \left(1-q^{n}\right)=-\frac{\pi^{2}}{6 s}-\frac{1}{2} \log (s)+\frac{1}{2} \log (2 \pi)-\frac{s}{24}+O\left(s^{M}\right)
$$

for any $M>0$. Additionally, by Lemma 5.2, we have

$$
\begin{aligned}
\sum_{n=1}^{\infty} \log \left(1-q^{n}\right)= & \int_{0}^{\infty} \log \left(1-e^{-x s}\right) d x-\int_{0}^{1 / 2} \log \left(1-e^{-x s}\right) d x \\
& -s \int_{1 / 2}^{\infty} \frac{e^{-x s}}{1-e^{-x s}}\left([x]-x+\frac{1}{2}\right) d x .
\end{aligned}
$$

Noting that $\int_{0}^{\infty} \log \left(1-e^{-x s}\right) d x=-\pi^{2} / 6 s$ and $\int_{0}^{1 / 2} \log \left(1-e^{-x s}\right) d x=$ $(1 / 2) \log (s)+\int_{0}^{1 / 2} \log (x) d x+O(s)$ we may conclude that

$$
-\int_{0}^{1 / 2} \log (x) d x-s \int_{1 / 2}^{\infty} \frac{e^{-x s}}{1-e^{-x s}}\left([x]-x+\frac{1}{2}\right) d x=\frac{1}{2} \log (2 \pi)+O(s) .
$$

Following the notation of $[9$, Section 3$]$ we define

$$
g_{k}(x s)=-\log \left(f_{k}\left(e^{-x s}\right)\right) .
$$


By the first line of Lemma 5.2,

$\sum_{n=1}^{\infty} g_{k}(n s)=\int_{0}^{\infty} g_{k}(x s) d x-\int_{0}^{1 / 2} g_{k}(x s) d x-s \int_{1 / 2}^{\infty} g_{k}^{\prime}(x s)\left([x]-x+\frac{1}{2}\right) d x$.

Theorem 1 of [17] gives $\int_{0}^{\infty} g_{k}(x s) d x=(1 / s)\left(\pi^{2} / 3 k(k+1)\right)$. Lemma 2.2 gives that for $s x \ll 1$

$$
g_{k}(x s)=-\log \left(f_{k}\left(e^{-x s}\right)\right)=-\frac{1}{k} \log (x s)-\frac{1}{k}(x s)^{1 / k}+O\left((x s)^{2 / k}\right) .
$$

Therefore, we have

$$
-\int_{0}^{1 / 2} g_{k}(x s) d x=\frac{1}{2 k} \log (s)-\frac{1}{k} \int_{0}^{1 / 2} \log (x) d x+O\left(s^{1 / k}\right) .
$$

Let $M=\left\lfloor s^{-1 / k}\right\rfloor$. Then we have by the second line of Lemma 5.2 that

$$
\begin{aligned}
s \int_{M+1 / 2}^{\infty} g_{k}^{\prime}(x s)\left([x]-x+\frac{1}{2}\right) d x & =\frac{s^{2}}{2} \int_{M+1 / 2}^{\infty} g_{k}^{\prime \prime}(x s)\left([x]-x+\frac{1}{2}\right)^{2} d x \\
& \ll s \int_{M s}^{\infty} g_{k}^{\prime \prime}(w) d w \ll M^{-1} \ll s^{1 / k}
\end{aligned}
$$

where we use $g^{\prime}(M s)=O_{k}(1 / M s)$ (see, for instance, [9, Lemma 3.1]).

To estimate the integral of $g_{k}^{\prime}$ from $1 / 2$ to $M+1 / 2$, we take the logarithmic derivative of $f_{k}\left(e^{-w}\right)^{k+1}-f_{k}\left(e^{-w}\right)^{k}=e^{-w(k+1)}-e^{-w k}$ to obtain

$$
g_{k}^{\prime}(w)=1-\frac{1}{k} \frac{e^{-w}}{e^{-w}-1}+\frac{1}{k} e^{-w} \frac{f_{k}^{\prime}\left(e^{-w}\right)}{1-f_{k}\left(e^{-w}\right)} .
$$

Therefore,

$$
\begin{aligned}
& s \int_{1 / 2}^{M+1 / 2} g_{k}^{\prime}(x s)\left([x]-x+\frac{1}{2}\right) d x \\
& =-\frac{s}{k} \int_{1 / 2}^{M+1 / 2} \frac{e^{-x s}}{1-e^{-x s}}\left([x]-x+\frac{1}{2}\right) d x \\
& \quad+\frac{s}{k} \int_{1 / 2}^{M+1 / 2} e^{-x s} \frac{f_{k}^{\prime}\left(e^{-x s}\right)}{1-f_{k}\left(e^{-x s}\right)}\left([x]-x+\frac{1}{2}\right) d x
\end{aligned}
$$


Observe that we have

$$
\begin{aligned}
& \int_{1 / 2}^{M+1 / 2} \frac{e^{-x s}}{1-e^{-x s}}\left([x]-x+\frac{1}{2}\right) d x \\
& \quad=\int_{1 / 2}^{\infty} \frac{e^{-x s}}{1-e^{-x s}}\left([x]-x+\frac{1}{2}\right) d x-\int_{M+1 / 2}^{\infty} \frac{e^{-x s}}{1-e^{-x s}}\left([x]-x+\frac{1}{2}\right) d x \\
& \quad=\int_{1 / 2}^{\infty} \frac{e^{-x s}}{1-e^{-x s}}\left([x]-x+\frac{1}{2}\right) d x+\frac{s}{2} \int_{M+1 / 2}^{\infty} \frac{e^{-x s}}{\left(1-e^{-x s}\right)^{2}}\left([x]-x+\frac{1}{2}\right)^{2} d x \\
& \quad=\int_{1 / 2}^{\infty} \frac{e^{-x s}}{1-e^{-x s}}\left([x]-x+\frac{1}{2}\right) d x+O\left(e^{-M s}\right)
\end{aligned}
$$

Additionally, integrating by parts, as in Lemma 5.2, gives

$$
\begin{aligned}
s \int_{1 / 2}^{M+1 / 2} & e^{-x s} \frac{f_{k}^{\prime}\left(e^{-x s}\right)}{1-f_{k}\left(e^{-x s}\right)}\left([x]-x+\frac{1}{2}\right) d x \\
& \left.\ll s \cdot \frac{e^{-x s} f_{k}^{\prime}\left(e^{-x s}\right)}{1-f_{k}\left(e^{-x s}\right)}\right|_{1 / 2} ^{M+1 / 2} \ll_{k} s^{1 / k}\left(1+M^{-(k-1) / k}\right)
\end{aligned}
$$

where we have used that monotonicity of $\log \left(1-f_{k}(w)\right)$ and $f_{k}^{\prime}(z)=O\left(z^{(1-k) / k}\right)$ for $z$ near 0. Returning to (5.5) and using (5.3) and (5.7)-(5.10)

$$
\begin{aligned}
- & \frac{1}{k} \int_{0}^{1 / 2} \log (x) d x-s \int_{1 / 2}^{\infty} g_{k}^{\prime}(x s)\left([x]-x+\frac{1}{2}\right) d x \\
= & \frac{1}{k}\left(-\int_{0}^{1 / 2} \log (x) d x-s \int_{1 / 2}^{\infty} \frac{e^{-x s}}{1-e^{-x s}}\left([x]-x+\frac{1}{2}\right) d x\right) \\
& +O\left(s^{1 / k}+M^{-1}\right) \\
= & \frac{1}{2 k} \log (2 \pi)+O\left(s^{1 / k}\right) .
\end{aligned}
$$

Finally, this together with (5.5) and (5.6) gives the result.

\section{Proof of Theorem 1.4}

In this section, we prove Theorem 1.4 and thus Theorem 1.2.

Proof of Theorem 1.4. We have $G_{k}(q)=\mathbf{e}^{T} \prod_{n=N+1}^{\infty} m\left(q^{n}\right) \cdot \prod_{n=1}^{N} m\left(q^{n}\right) \mathbf{e}_{1}$. It follows from Theorem 4.1, Proposition 3.4, and Theorems 4.4 and 5.1 that for 
appropriate $N$,

$$
\begin{aligned}
G_{k}\left(e^{-s}\right)= & \frac{1}{k} \exp \left(\frac{\pi^{2}}{6 s}\left(1-\frac{2}{k(k+1)}\right)\right. \\
& \left.+O\left(N^{-(k+1) / k} s^{-1 / k}+s N^{2}+s^{2 / k} N^{(k+2) / k}+N^{-1}\right)\right) .
\end{aligned}
$$

Setting $N=\left\lfloor s^{-3 /(2 k+3)}\right\rfloor$ yields the result.

\section{Proof of Theorem 1.8}

In this section, we apply a result of Ingham [18] to deduce the asymptotics for $p_{k}(n)$ from the asymptotics of $G_{k}(q)$ as $q \rightarrow 1$. In particular, we have the following result which is a special case of [18, Theorem 1] and is given as [12, Theorem 4.1].

THEOREM 7.1 (Ingham). Let $f(z)=\sum_{n=0}^{\infty} a(n) z^{n}$ be a power series with real nonnegative coefficients and radius of convergence equal to 1 . If there exists $A>0, \lambda, \alpha \in \mathbb{R}$ such that

$$
f(z) \sim \lambda(-\log (z))^{\alpha} \exp \left(-\frac{A}{\log (z)}\right)
$$

as $z \rightarrow 1^{-}$, then

$$
\sum_{m=0}^{n} a(m) \sim \frac{\lambda}{2 \sqrt{\pi}} \frac{A^{\alpha / 2-1 / 4}}{n^{\alpha / 2+1 / 4}} \exp (2 \sqrt{A n})
$$

as $n \rightarrow \infty$.

Proof of Theorem 1.8. By [17, Lemma 10]

$$
(1-q) G_{k}(q)=\sum_{n=0}^{\infty}\left(p_{k}(n)-p_{k}(n-1)\right) q^{n}
$$

has nonnegative coefficients. Applying Theorems 1.4 and 7.1 gives the result.

\section{Acknowledgements}

The authors thank Stanford University and the National Science Foundation for support during the preparation of this work. The authors are grateful to the 
anonymous referees and Karl Mahlburg for correcting errors in earlier versions of the manuscript and providing keen insights and useful feedback.

The authors were partially supported by NSF Mathematical Sciences Postdoctoral Fellowships during the preparation of this work.

\section{References}

[1] M. Aizenman and J. Lebowitz, 'Metastability effects in bootstrap percolation', J. Phys. (A) 21 (1988), 3801-3813.

[2] G. E. Andrews, 'Some new partition theorems', J. Combin. Theory 2 (1967), 431-436.

[3] G. E. Andrews, 'Partitions with short sequences and mock theta functions', Proc. Natl. Acad. Sci. USA 102 (2005), 4666-4671.

[4] G. E. Andrews, H. Eriksson, F. Petrov and D. Romik, 'Integrals, partitions and MacMahon's theorem', J. Combin. Theory (A) 114 (2007), 545-555.

[5] G. E. Andrews and R. P. Lewis, 'An algebraic identity of F. H. Jackson and its implications for partitions', Discrete Math. 232 (2001), 77-83.

[6] R. J. Baxter, Exactly Solved Models in Statistical Mechanics, (Academic Press, London and New York, 1982).

[7] K. Bringmann, B. Kane, D. Parry and R. C. Rhoades, 'On the Andrews-Zagier asymptotics for partitions without sequences', Adv. Math. 309 (2017), 436-451.

[8] K. Bringmann and K. Mahlburg, 'An extension of the Hardy-Ramanujan Circle Method and applications to partitions without sequences', Amer. J. Math. 133 (2011), 1151-1178.

[9] K. Bringmann and K. Mahlburg, 'Improved bounds on metastability thresholds and probabilities for generalized bootstrap percolation', Trans. Amer. Math. Soc. 364 (2012), 3829-3859.

[10] K. Bringmann, K. Mahlburg and A. Mellit, 'Convolution bootstrap percolation models, Markov-type stochastic processes, and mock theta functions', Int. Math. Res. Not. (2013), 971-1013.

[11] K. Bringmann, K. Mahlburg and K. Nataraj, 'Distinct parts partitions without sequences', Electronic J. Combin. 22 (2015), Paper 3.3, 13 pp.

[12] K. Bringmann, A. Holroyd, K. Mahlburg and M. Vlasenko, ' $k$-run overpartitions and mock theta functions', Q. J. Math. 64 (2013), 1009-1021.

[13] B. Fristedt, 'The structure of random partitions of large integers', Trans. Amer. Math. Soc. 337(2) 703-735.

[14] J. Gravner and A. Holroyd, 'Slow convergence in bootstrap percolation', Ann. Appl. Probab. 18 (2008), 909-928.

[15] D. J. Griffiths, Introduction to Quantum Mechanics, second edition, (Pearson Education Inc., Upper Saddle River NJ, 2005).

[16] A. E. Holroyd, 'Sharp metastability threshold for two-dimensional bootstrap percolation', Probab. Theory Related Fields 125 (2003), 195-224.

[17] A. E. Holroyd, T. M. Liggett and D. Romik, 'Integrals, partitions, and cellular automata', Trans. Amer. Math Soc. 356 (2004), 3349-3368.

[18] A. Ingham, 'A Tauberian theorem for partitions', Ann. of Math. (2) 42 (1941), 1075-1090.

[19] A. Knopfmacher and A. O. Munagi, 'Successions in integer partitions', Ramanujan J. 18 (2009), 239-255.

[20] P. A. MacMahon, Combinatorial Analysis, Vol. 2, (Cambridge University Press, New York, 1916), Reprinted Dover. 
[21] K. Ono, 'Unearthing the visions of a master: harmonic Maass forms and number theory', in Current Developments in Mathematics (Int. Press, Somerville, MA, 2009), 347-454.

[22] D. Zagier, 'Ramanujan's mock theta functions and their applications [d'aprés Zwegers and Bringmann-Ono]', in Sém. Bourbaki (2007/2008), Astérisque, No. 326, Exp. No. 986, vii-viii (2010), 143-164.

[23] D. Zagier, private communication.

[24] S. Zwegers, 'Mock theta functions', PhD Thesis (Advisor: D. Zagier), Universiteit Utrecht, (2002). 